JOURNAL OF THE AMERICAN MATHEMATICAL SOCIETY

Volume 12, Number 4, Pages 981-1015

S 0894-0347(99)00309-4

Article electronically published on June 7, 1999

\title{
MULTIDIMENSIONAL VAN DER CORPUT AND SUBLEVEL SET ESTIMATES
}

\author{
ANTHONY CARBERY, MICHAEL CHRIST, AND JAMES WRIGHT
}

\section{INTRODUCTION}

If a function has a large derivative, then it changes rapidly, and so spends little time near any particular value. This paper is devoted to quantifying that principle for functions of several variables, particularly as it pertains to two problems in harmonic analysis. Along the way we shall encounter diverse problems and techniques, and shall be led to issues distinctly combinatorial in nature.

We begin by reviewing two well-known questions in one-dimensional analysis. Suppose that $u$ is a (smooth) real-valued function on the real line $\mathbb{R}$ such that for some $k \in \mathbb{N}, u^{(k)}(t) \geq 1$ for all $t \in \mathbb{R}$. Here, and in what follows, $u^{(k)}$ denotes the $k$ 'th derivative of $u$.

a) How small are the sublevel sets $\{t \in \mathbb{R}:|u(t)| \leq \alpha\}$ for small $\alpha$ ? In particular, at what rate does the Lebesgue measure $|\{t \in \mathbb{R}:|u(t)| \leq \alpha\}|$ tend to zero as $\alpha \rightarrow 0$ ?

b) How quickly does the oscillatory integral $I(\lambda)=\int_{a}^{b} e^{i \lambda u(t)} d t$ tend to zero as $\lambda \rightarrow \infty$ ?

Answers are given by the following two results. The first is known as van der Corput's lemma; see, for example, [S].

Lemma 1.1. a) Suppose $k \geq 2$. If $u^{(k)} \geq 1$, then $|I(\lambda)| \leq C_{k} /|\lambda|^{\frac{1}{k}}$ where $C_{k}$ is an absolute constant depending only upon $k$.

b) There is no constant $C$ such that if $u^{\prime} \geq 1$, then $|I(\lambda)| \leq C /|\lambda|$.

c) If $u^{\prime} \geq 1$ and if in addition $u^{\prime}$ is monotonic, then $|I(\lambda)| \leq C_{1} /|\lambda|$ where $C_{1}$ is an absolute constant.

Lemma 1.2. For each $k \geq 1$, there exists a finite absolute constant $C_{k}$ such that for any function satisfying $u^{(k)}(x) \geq 1$ for all $x$,

$$
|\{t:|u(t)| \leq \alpha\}| \leq C_{k} \alpha^{1 / k} .
$$

The estimates in Lemmas 1.1 and 1.2 are uniform over classes of functions $u$ whose $k$ 'th derivatives are bounded below; they are independent of any upper bounds for higher order derivatives of $u$. Our chief goal is to establish analogous uniform estimates in higher dimensions.

Received by the editors June 24, 1998.

1991 Mathematics Subject Classification. Primary 42B10; Secondary 26D10, 05D99.

Key words and phrases. Oscillatory integrals, sublevel sets, van der Corput lemma, combinatorics.

This work was partially supported by EPSRC grants GR/L10024 and GR/L78574 (Carbery), NSF grant DMS 9623007 (Christ), ARC grants (Wright), and MSRI. 
In dimensions greater than one, satisfactory results are generally valid only for functions $u$ defined on suitable bounded subsets of $\mathbb{R}^{n}$, rather than on the whole space. For simplicity we work on the unit cube $Q=Q^{n}=[0,1]^{n}$ (or on $Q^{n^{\prime}} \times Q^{n^{\prime \prime}}$ ), but as the arguments will demonstrate, all results hold for arbitrary convex subsets of $\mathbb{R}^{n}$ (or Cartesian products of two convex sets) with uniformly bounded diameters, with uniform bounds in the conclusions. Two of our principal results are as follows:

Theorem 1.3. For any multi-index $\beta$ there exist $\varepsilon>0$ and $C<\infty$, depending only on $\beta$ and on $n$, such that for any integrable $u: Q \rightarrow \mathbb{R}$ that satisfies $D^{\beta} u \geq 1$ on $Q$ in the sense of distributions, one has for each $\alpha>0$ the sublevel set estimate

$$
|\{x \in Q:|u(x)| \leq \alpha\}| \leq C \alpha^{\varepsilon} .
$$

Theorem 1.4. Let $\beta=\left(\beta_{1}, \ldots, \beta_{n}\right) \neq 0$ be a multi-index, and suppose that at least one of its entries $\beta_{j}$ is greater than or equal to two. Then there exist $\varepsilon>0$ and $C<\infty$, depending only on $\beta$ and on $n$, such that for any integrable $u: Q \rightarrow \mathbb{R}$ satisfying $D^{\beta} u \geq 1$ on $Q$ in the sense of distributions, for all $\lambda \in \mathbb{R}$, the oscillatory integral $I(\lambda)=\int_{Q} e^{i \lambda u(x)} d x$ satisfies

$$
|I(\lambda)| \leq C|\lambda|^{-\varepsilon} .
$$

Throughout the discussion, our aim is to obtain estimates entirely independent of any other (quantitative) assumptions concerning $u$. That is, both $\varepsilon$ and $C$ should depend only on $\beta$ and the dimension $n$. Previous results in dimensions $>1$ - where the constant $C$ depends also on $\|u\|_{C^{|\beta|+1}}$ for example, see [S] have lacked this uniformity. Part of our motivation is the quest for a robust theory stable under perturbations. In many problems, particularly those concerning singular integral operators, scaling and normalisation play a central role, and families of functions $u$ may arise, which depend upon various parameters involved in the scaling; it is essential to obtain estimates independent of those parameters.

It would be equivalent to state Theorems 1.3 and 1.4 only for $C^{\infty}$ functions $u$, satisfying $D^{\beta} u \geq 1$ in the ordinary pointwise sense, with the upper bounds in the conclusions uniform over all such $u$. For a simple approximation argument based on convolution with approximate identities then yields the conclusion for arbitrary integrable functions satisfying the lower bound in the distribution sense.

We have been unable to obtain the seemingly natural exponent $\varepsilon=|\beta|^{-1}$ in Theorems 1.3 and 1.4. (The exponent $|\beta|^{-1}$ can be obtained if $C$ is allowed to depend on the $C^{|\beta|+1}$ norm of $u$.) For large multi-indices $\beta$, and for high dimensions, there is a large gap between our results and those counterexamples known to us.

What may be unexpected is that certain combinatorial problems should be central to our higher-dimensional analysis. As an example of such a problem, consider a matrix $\mathcal{M}=\left(m_{i, j}\right)$ where $1 \leq i, j \leq N$, each of whose entries $m_{i, j}$ is equal either to 1 or to 0 . By a $2 \times 2$ submatrix we mean any matrix $\left(m_{i(p), j(q)}\right)$ where $p, q \in\{1,2\}$ and $1 \leq i(p), j(q) \leq N$ for each $p, q$. In particular, we allow $i(1)=i(2)$ and/or $j(1)=j(2)$. By the area of such a submatrix we mean the quantity $(|i(1)-i(2)|+1)(|j(1)-j(2)|+1)$.

Problem 1.1. Does there exist $\varepsilon>0$ such that for every $M, N \geq 1$, any $N \times N$ matrix $\mathcal{M}$ which has at least $M$ entries equal to 1 has a $2 \times 2$ submatrix all of whose entries equal 1, whose area is at least $\varepsilon M^{2} / N^{2}$ ?

\footnotetext{
${ }^{1} \mathrm{By}$ an integrable function we mean a Lebesgue measurable function in $L^{1}(Q)$.
} 
This is the simplest of a hierarchy of related problems that arise, in continuous rather than discrete formulations, in our analysis. To the best of our knowledge it remains open; our rather naive methods yield the existence of a submatrix with area $\geq \varepsilon M^{2} / N^{2} \log (N)$.

Our methods apply in an operator-theoretic setting, that is, to

$$
S_{\alpha} f\left(x^{\prime}\right)=\int \chi_{\alpha}\left(x^{\prime}, x^{\prime \prime}\right) f\left(x^{\prime \prime}\right) d x^{\prime \prime} \quad \text { and } \quad T_{\lambda} f\left(x^{\prime}\right)=\int e^{i \lambda u\left(x^{\prime}, x^{\prime \prime}\right)} f\left(x^{\prime \prime}\right) d x^{\prime \prime}
$$

where $x=\left(x^{\prime}, x^{\prime \prime}\right) \in \mathbb{R}^{n^{\prime}+n^{\prime \prime}}$, and $\chi_{\alpha}$ is the characteristic function of the set where $|u(x)|<\alpha$. Here one seeks decay estimates for the operator norms from $L^{p}\left(Q^{n^{\prime \prime}}\right)$ to $L^{q}\left(Q^{n^{\prime}}\right)$ as $\alpha \rightarrow 0$ and $|\lambda| \rightarrow \infty$, which depend only upon the basic hypothesis $D^{\beta} u \geq 1$ on $Q$. This is achieved in Theorems 3.16 and 4.13 , though with seemingly poor decay exponents. ${ }^{2}$ (Of course since the $T_{\lambda}$ problem specializes, for particular choices of $u$, to some of the leading unsolved problems of harmonic analysis, this lack of optimality is no surprise.) In the special case where $n=2$ and $\beta=(1, k)$ with $k \geq 2$, we are able to obtain the sharp $L^{2}-L^{2}$ result for $T_{\lambda}$; see Theorem 4.8.

These various problems are interrelated. The usual proof of the van der Corput Lemma 1.1 uses the sublevel set estimate of Lemma 1.2 together with a simple integration by parts argument. On the other hand, estimates for oscillatory integrals imply estimates for sublevel sets, via an old device: writing $\phi(u(x))=$ $\int_{\mathbb{R}} e^{i \lambda u(x)} \hat{\phi}(\lambda) d \lambda$, estimates on $I(\lambda)$ or $\left\|T_{\lambda}\right\|$ translate directly to estimates on $\int \phi(u(x)) d x$ or the operator whose kernel is $\phi(u(x))$. In particular, choosing $\phi(t)=$ $\psi(t / \alpha)$ we see that $I(\lambda)=O\left(|\lambda|^{-\delta}\right)$ implies $|\{x:|u(x)| \leq \alpha\}| \leq C \alpha^{\delta}$ and that $\left\|T_{\lambda}\right\|=O\left(|\lambda|^{-\delta}\right)$ implies $\left\|S_{\alpha}\right\|=O\left(\alpha^{\delta}\right)$. In [C2] sublevel set estimates appeared as a central part of a mechanism devised to substitute for oscillatory integral estimates, where no reduction to the latter was readily available. Finally, the problem addressed by Theorem 1.3 is the special case $p=\infty, q=1$ of the problem for $S_{\alpha}$ and the problem for $I(\lambda)$ is a special case of the instance $p=\infty, q=1$ of the problem for $T_{\lambda}$, and can in fact (see Proposition 4.1) be used to solve that problem.

Motivated in part by the case $k=1$ of van der Corput's lemma, we also explore what happens if supplementary higher order "convexity" type hypotheses are imposed on $u$; in the settings of Theorems 1.3 and 1.4 we are then able to recover the natural decay rates $O\left(\alpha^{-1 /|\beta|}\right)$ and $O\left(\lambda^{-1 /|\beta|}\right)$, respectively. See Sections 3 and 4 for proofs, and Section 7 for an application. When $n=2$ we likewise obtain the optimal $L^{p}-L^{q}$ mapping properties of $S_{\alpha}$ under additional "convexity" hypotheses; see Theorem 3.13.

The layout of the paper is as follows. In Section 2 we give two basic real variable lemmas, and deduce versions of Lemmas 1.1 and 1.2. In Section 3 we address the sublevel set and $S_{\alpha}$ problems, while the corresponding $I(\lambda)$ and $T_{\lambda}$ problems are treated in Section 4. Many of the results of Section 3 for sublevel set estimates could alternatively be deduced from the results of Section 4, as remarked above, but with inferior exponents. The possible replacement of $D^{\beta}$ by other partial differential operators such as the Laplacian is discussed briefly in Section 5. Section 6 contains remarks concerning the combinatorial nature of our problems. Section 7 examines the special case where $u$ is a polynomial of bounded degree, while Section 8 contains

\footnotetext{
${ }^{2}$ Better or optimal decay estimates for $T_{\lambda}$ — but requiring more assumptions concerning $u$ such as quantitative smoothness — have been obtained by Phong and Stein [PS2] and by Seeger [Se2] in the case $n=2$.
} 
some applications; in both sections our basic hypothesis $D^{\beta} u \geq 1$ arises naturally. In Section 9 we briefly discuss the sublevel set problem, under the hypothesis that $\partial^{\beta} u$ is not merely bounded below, but is $C^{\infty}$. It might well also be natural to posit lower bounds on several different partial derivatives of $u$, in the spirit of work of Phong and Stein, but that problem is not considered in this paper.

All our results concerning $I(\lambda)$ and $T_{\lambda}$ are equally valid for $\int e^{i \lambda u(x)} \phi(x) d x$ and $\int e^{i \lambda u\left(x^{\prime}, x^{\prime \prime}\right)} f\left(x^{\prime \prime}\right) \phi\left(x^{\prime}, x^{\prime \prime}\right) d x^{\prime \prime}$, where $\phi$ is a fixed smooth function of compact support in $Q$; of course now the constants will depend (in an uninteresting way) upon $\phi$ and some of its derivatives. Moreover all our results remain valid when $u$ is merely an integrable function satisfying $D^{\beta} u \geq 1$ in the sense of distributions.

Concerning notation, the $L^{p}-L^{q}$ operator norm of an operator $P$ is denoted by $\|P\|_{p \mapsto q}$, or $\|P\|_{L^{p} \rightarrow L^{q}}$, or simply $\|P\|$ when the context is clear. $C$ denotes a generic constant depending only on the parameters made explicit during any argument; the precise value of $C$ may vary from line to line. The expression $A \sim B$ means that $A / B$ is bounded away from zero and infinity. $\mathbb{N}$ denotes the set $\{0,1,2, \ldots\}$. We write $\wedge$ for 'min', $\vee$ for ' $m a x$ ', and $\# E$ for the cardinality of a set $E$.

\section{The OnE-DIMENSIONAL CASE REVISITED}

As stated in the introduction, in order to prove our main results, in which $u$ is an integrable function satisfying $\partial^{\beta} u \geq 1$ in the sense of distributions, it suffices to prove them for $C^{\infty}$ functions satisfying such a lower bound, so long as the bounds obtained are uniform over the class of all such $u$. To justify this reduction, fix a real-valued, nonnegative auxiliary function $\eta \in C_{0}^{\infty}\left(\mathbb{R}^{n}\right)$ supported in the unit ball centered at the origin, satisfying $\int \eta=1$. Set $\eta_{t}(x)=t^{-n} \eta(x / t)$, and $u^{(t)}(x)=u * \eta_{t}(x)$. Then $u$ satisfies the bound $\partial^{\beta} u \geq 1$ on the unit cube $Q$, in the sense of distributions, if and only if each $u^{(t)}$ satisfies the same bound in the pointwise sense, on the domain $Q_{t}=\{x \in Q$ : $\operatorname{dist}(x, \partial Q)<t\}$. Likewise all the oscillatory integral and sublevel set estimates considered in this paper hold for $u$, if and only if they hold uniformly for all $u^{(t)}$ as $t \rightarrow 0$, with $Q$ replaced by $Q_{t}$. Consequently we will assume without comment in proofs throughout the paper that $u \in C^{\infty}$.

In this section we give versions of the one-dimensional van der Corput lemma and the corresponding sublevel set estimate which are due to Arhipov, Karacuba and Cubarikov [AKC]. These more precise formulations appear not to be well-known and so we have included them for completeness.

Proposition 2.1. If $u$ is an integrable, real-valued function satisfying $u^{(k)}(t) \geq 1$ on $\mathbb{R}$ in the sense of distributions, then

$$
|\{t:|u(t)| \leq \alpha\}| \leq(2 e)((k+1) !)^{1 / k} \alpha^{1 / k} .
$$

Proposition 2.2. There exists an absolute constant $C$ so that for any $a<b$, any $k \geq 2$, and any integrable real-valued function satisfying $u^{(k)} \geq 1$ in the sense of distributions, for every $\lambda$,

$$
\left|\int_{a}^{b} e^{i \lambda u(t)} d t\right| \leq C k|\lambda|^{-1 / k}
$$

Remark. That the linear growth in $k$ of the constants in the above propositions is optimal may be seen by examining the function $u(t)=t^{k} / k$ !. For an application see Section 7 . 
Before proving the propositions we give two lemmas, upon which our whole analysis in both one and several variables rests. The first is a generalisation of the classical mean-value theorem and can be found in texts on numerical analysis, for example [IK], p.189ff. It employs Lagrange interpolation for polynomials. For the convenience of the reader we include a proof.

Lemma 2.3. Let $k \geq 1$, suppose that $a_{1}, \ldots, a_{k+1} \in \mathbb{R}$ are distinct, that $f$ is $a$ $C^{k-1}$ mapping from the closed convex hull $\overline{c v x}\left\{a_{1}, \ldots, a_{k+1}\right\}$ to $\mathbb{R}$, and that $f$ is $k$ times differentiable on $\operatorname{cvx}\left\{a_{1}, \ldots, a_{k+1}\right\}$. Then there exists $a \zeta \in \operatorname{cvx}\left\{a_{1}, \ldots, a_{k+1}\right\}$ such that

$$
\sum_{m=1}^{k+1} c_{m} f\left(a_{m}\right)=f^{(k)}(\zeta)
$$

where $c_{m}= \pm k ! \prod_{\ell: \ell \neq m}\left|a_{\ell}-a_{m}\right|^{-1}$

Here $c v x$ and $\overline{c v x}$ denote respectively the open convex hull of a set and its closure.

Proof. Let $\phi(x)=f(x)-A x^{k}+p_{k-1}(x)$ where $p_{k-1}$ is some polynomial of degree $k-1$ and $A \in \mathbb{R}$. We assert that we can choose $p_{k-1}$ and $A$ so that $\phi\left(a_{m}\right)=$ $0,1 \leq m \leq k+1$. If so, then by Rolle's theorem $\phi^{(k)}$ vanishes somewhere in $\operatorname{cv} x\left\{a_{1}, \ldots, a_{k+1}\right\}$. So there is a $\zeta \in \operatorname{cvx}\left\{a_{1}, \ldots, a_{k+1}\right\}$ such that $f^{(k)}(\zeta)=k ! A$. So it remains to find the $A$ and $p_{k-1}$ such that $\phi\left(a_{m}\right)=0$ for all $m$. Clearly this holds if and only if the coefficients $b_{0}, \ldots, b_{k-1}$ of $p_{k-1}$ and $A$ satisfy the matrix equation

$$
V_{k+1}\left(a_{1}, \ldots, a_{k+1}\right)\left(\begin{array}{c}
b_{0} \\
\vdots \\
b_{k-1} \\
-A
\end{array}\right)=-\left(\begin{array}{c}
f\left(a_{1}\right) \\
\vdots \\
\vdots \\
f\left(a_{k+1}\right)
\end{array}\right)
$$

where $V_{k+1}$ is the Vandermonde matrix in $\left\{a_{1}, \ldots, a_{k+1}\right\}$ with 1 's down the first column. This equation clearly has a unique solution when the $a_{m}$ are all distinct, and Cramér's rule shows that

$$
A=\sum_{m=1}^{k+1} \pm \frac{\operatorname{det} V_{k}\left(a_{1}, \ldots, \hat{a}_{m}, \ldots, a_{k+1}\right)}{\operatorname{det} V_{k+1}\left(a_{1}, \ldots, a_{k+1}\right)} f\left(a_{m}\right)
$$

where the circumflex in the $V_{k}$ in the numerator indicates omission of $a_{m}$. By the formula for the Vandermonde determinant the coefficient of $f\left(a_{m}\right)$ in this expression is $\pm \prod_{\ell: \ell \neq m}\left|a_{\ell}-a_{m}\right|^{-1}$, as desired.

Lemma 2.4. Let $E \subseteq \mathbb{R},|E|>0$, and $k \in \mathbb{N}$. Then there exist $a_{0}, a_{1}, \ldots, a_{k} \in E$ such that for all $\ell=0,1, \ldots, k$,

$$
\prod_{j: j \neq \ell}\left|a_{j}-a_{\ell}\right| \geq(|E| / 2 e)^{k}
$$

Proof. Since an arbitrary measurable subset of $\mathbb{R}$ can be mapped to an interval by a measure-preserving transformation which does not increase distances, the worst case clearly occurs when $E$ is an interval. Scaling permits us to assume $E=[0,1]$. Taking $a_{j}=j / k$, we see that the value of $\ell$ giving the least value of the left side 
in the proposed inequality is $k / 2$ when $k$ is even and $(k \pm 1) / 2$ when $k$ is odd. For even $k$, the left-hand side for $\ell=k / 2$ is

$$
\left\{\frac{1}{k} \cdot \frac{2}{k} \ldots \frac{(k / 2)}{k}\right\}^{2}=\left\{\frac{(k / 2) !}{(k / 2)^{\frac{k}{2}} 2^{\frac{k}{2}}}\right\}^{2} \geq(2 e)^{-k} .
$$

For odd $k$, the left-hand side for $\ell=(k \pm 1) / 2$ is

$$
\left\{\frac{1}{k} \cdot \frac{2}{k} \ldots \frac{(k-1) / 2}{k}\right\}^{2} \frac{k+1}{2 k} \geq(2 e)^{-k}
$$

also.

Proof of Proposition 2.1. Let $E=\{t:|u(t)| \leq \alpha\}$. If $|E|>0$, there exist, by Lemma 2.4, $a_{0}, \ldots, a_{k} \in E$ so that for all $\ell$

$$
|E|^{k} \leq(2 e)^{k} \prod_{j: j \neq \ell}\left|a_{j}-a_{\ell}\right|
$$

On the other hand, Lemma 2.3 implies that for some $\zeta \in \operatorname{cv} x\left\{a_{0}, \ldots, a_{k}\right\}$,

$$
\begin{aligned}
u^{(k)}(\zeta) & =k ! \sum \pm u\left(a_{j}\right) \prod_{l: j \neq \ell}\left|a_{j}-a_{\ell}\right|^{-1} \\
& \leq(k+1) ! \max \left|u\left(a_{j}\right)\right|(2 e)^{k}|E|^{-k}
\end{aligned}
$$

The bound $u^{(k)}(t) \geq 1$ now implies $|E|^{k} \leq(k+1) !(2 e)^{k} \alpha$, as desired.

Proof of Proposition 2.2. For a parameter $\beta$ to be determined we split the integral as $I+I I$, where $I$ is the integral over the region where $\left|u^{\prime}(x)\right| \leq \beta$. To estimate $I$ we apply Proposition 2.1 to the function $v=u^{\prime}$ which satisfies $v^{(k-1)} \geq 1$ and obtain $|\{|v| \leq \beta\}| \leq C k \beta^{1 /(k-1)}$. For $I I$, we observe that $u^{(k)} \geq 1$ means that $\left\{\left|u^{\prime}\right|>\beta\right\}$ is the union of at most $O(k)$ intervals on each of which $u^{\prime}$ is monotone, and on each of which we may integrate by parts in the usual fashion to obtain a bound which is $O(1 /|\lambda| \beta)$. Thus $|I I| \leq C k /|\lambda| \beta$. Optimising in $\beta\left(\beta \approx|\lambda|^{-(k-1) / k}\right)$ yields the result.

\section{Operators ASSOCIATED TO SUBlevel SETS}

Throughout the paper we use the following notation.

Definition. $\mathcal{U}_{\beta}=\mathcal{U}_{\beta}(Q)$ denotes the set of all $C^{\infty}$ real-valued functions $u \in$ $C^{\infty}(Q)$ that satisfy

$$
D^{\beta} u \geq 1
$$

at every point of $Q$. $\tilde{\mathcal{U}}_{\beta}$ denotes the set of all integrable real-valued functions $u$ that satisfy (3.1) on $Q$, in the sense of distributions.

$Q=Q^{n}$ denotes the unit cube in $\mathbb{R}^{n}$, and $\beta=\left(\beta_{1}, \ldots, \beta_{n}\right)$ is a multi-index for which each $\beta_{j} \in\{0,1,2, \ldots\}$. When $n=2$, we will often write $\mathcal{U}_{j, k}$ rather than $\mathcal{U}_{(j, k)}$.

Set

$$
E_{\alpha}=\{x \in Q:|u(x)| \leq \alpha\}
$$


and, for $n^{\prime}+n^{\prime \prime}=n\left(1 \leq n^{\prime} \leq n-1\right)$, define the operators $S_{\alpha}$ taking functions on $Q^{n^{\prime \prime}}$ to functions on $Q^{n^{\prime}}$ by

$$
S_{\alpha} f\left(x^{\prime}\right)=\int_{Q^{n^{\prime \prime}}} \chi_{E_{\alpha}}(x) f\left(x^{\prime \prime}\right) d x^{\prime \prime}
$$

where $x \in Q^{n}$ is written as $x=\left(x^{\prime}, x^{\prime \prime}\right) \in Q^{n^{\prime}} \times Q^{n^{\prime \prime}}$. We examine the $L^{p}-L^{q}$ mapping properties of $S_{\alpha}$ as a function of the (small) parameter $\alpha$. The special case $p=\infty, q=1$ of this problem is of course equivalent to estimating $\left|E_{\alpha}\right|$.

3.1. The two-dimensional case. For $0<\alpha<1$ let $E_{\alpha}=\left\{(x, y) \in Q^{2}\right.$ : $|u(x, y)|<\alpha\}$ and rewrite $(3.2)$ as $S_{\alpha} f(x)=\int_{[0,1]} \chi_{E_{\alpha}}(x, y) f(y) d y$. We suppose $u \in \tilde{\mathcal{U}}_{j, k}$, that is, $u$ is integrable, real-valued in $Q$, and satisfies

$$
\frac{\partial^{d} u}{\partial x^{j} \partial y^{k}} \geq 1 \text { on } Q
$$

in the sense of distributions, where $d=j+k$ and $0 \leq j, k \leq d$. We investigate the $L^{p} \rightarrow L^{q}$ boundedness properties of $S_{\alpha}$ as a function of $\alpha$. We first give some lower bounds on $\left\|S_{\alpha}\right\|=\left\|S_{\alpha}\right\|_{L^{p} \rightarrow L^{q}}$ for particular $u$ satisfying (3.3).

Proposition 3.1. For each $j, k$ there exists $C<\infty$ such that the following hold:

(a) If $u(x, y)=-(x-y)^{d}$ with $d \geq 2$, then $\left\|S_{\alpha}\right\| \geq C \alpha^{1 / d}$.

(b) If $u(x, y)=x^{j} y^{k}$, then $\left\|S_{\alpha}\right\| \geq C \alpha^{1 / j q}$.

(c) If $u(x, y)=x^{j} y^{k}$, then $\left\|S_{\alpha}\right\| \geq C \alpha^{1 / k p^{\prime}}$.

Proof. (a) Let $f=\chi_{(0,1)}$; then $S_{\alpha} f$ is essentially the constant $\alpha^{1 / d}$ and thus $\left\|S_{\alpha}\right\| \geq$ $C \alpha^{1 / d}$.

(b) If $0<x<\alpha^{1 / j}, S_{\alpha} f(x)=\int_{0}^{1} f(y) d y$, and so $\left\|S_{\alpha}\right\| \geq C \alpha^{1 / j q}$.

(c) If $0<y<\alpha^{1 / k}, S_{\alpha}^{*} g(y)=\int_{0}^{1} g(x) d x$, and so $\left\|S_{\alpha}\right\|_{p \mapsto q}=\left\|S_{\alpha}^{*}\right\|_{q^{\prime}-p^{\prime}} \geq$ $C \alpha^{1 / k p^{\prime}}$.

Remark. For the choices of $u$ in the above proposition, one can easily show that $\left\|S_{\alpha}\right\| \leq C \alpha^{\frac{1}{d} \wedge \frac{1}{d}\left[1+\frac{1}{q}-\frac{1}{p}\right]}$ when $u(x, y)=(x-y)^{d}$ and $\left\|S_{\alpha}\right\| \leq C \alpha^{\frac{1}{j q} \wedge \frac{1}{k p^{\prime}}}$ when $u(x, y)=x^{j} y^{k}$.

Corollary 3.2. $\sup _{u \in \mathcal{U}_{j, k}}\left\|S_{\alpha}\right\| \geq C \alpha^{\frac{1}{d} \wedge \frac{1}{j q} \wedge \frac{1}{k p^{\prime}}}$.

Note that $1 / j q=1 / k p^{\prime}=1 / d$ precisely when $p=q=d / j$. So were we able to prove $\left\|S_{\alpha}\right\|_{L^{d / j} \rightarrow L^{d / j}} \leq C \alpha^{1 / d}$ we would have $\left\|S_{\alpha}\right\| \leq C \alpha^{\frac{1}{d} \wedge \frac{1}{j q} \wedge \frac{1}{k p^{\prime}}}$ for all $1 \leq p, q \leq$ $\infty$ by interpolation with trivial estimates. We now examine what upper bounds can be obtained for $\left\|S_{\alpha}\right\|$.

Proposition 3.3. If $j=0$ and $k=d$, then $\left\|S_{\alpha}\right\| \leq C \alpha^{1 / d p^{\prime}}$; if $j=d$ and $k=0$, then $\left\|S_{\alpha}\right\| \leq C \alpha^{1 / d q}$.

The proof is an easy consequence of the corresponding one-dimensional result; the details are left to the reader.

Theorem 3.4. There exists an absolute constant $C$ so that if $\partial^{2} u / \partial x \partial y \geq 1$ (i.e. $\left.u \in \tilde{\mathcal{U}}_{1,1}\right)$, then $\left\|S_{\alpha}\right\|_{L^{2} \rightarrow L^{2}} \leq C \alpha^{1 / 2} \log ^{1 / 2}(1 / \alpha)$ for $0<\alpha<1 / 2$. 
Corollary 3.5. There is an absolute constant $C$ so that for all $u \in \tilde{\mathcal{U}}_{1,1}$

$$
|\{(x, y) \in Q:|u(x, y)| \leq \alpha\}| \leq C \alpha^{1 / 2} \log ^{1 / 2}(1 / \alpha) .
$$

Thus we are missing the result for $\tilde{\mathcal{U}}_{1,1}$ suggested by Corollary 3.2 by a logarithmic factor; we do not know whether this factor is necessarily present.

We begin the proof of Theorem 3.4 with a lemma.

Lemma 3.6. Let $u \in \mathcal{U}_{1,1}, 0<\alpha<1 / 2, E=\{(x, y) \in Q:|u(x, y)| \leq \alpha\}$, and $E(y)=\{x:(x, y) \in E\}$. Then

$$
\left|E\left(y_{1}\right) \cap E\left(y_{2}\right)\right| \leq 4 \alpha /\left|y_{1}-y_{2}\right| .
$$

Proof. If $x \in E\left(y_{1}\right) \cap E\left(y_{2}\right)$, then $\left|u\left(x, y_{j}\right)\right| \leq \alpha$ for $j=1$ and 2 and so

$$
\left|u\left(x, y_{1}\right)-u\left(x, y_{2}\right)\right| \leq 2 \alpha .
$$

For $y_{1}, y_{2}$ fixed let $\psi(x)=u\left(x, y_{1}\right)-u\left(x, y_{2}\right)=\int_{y_{2}}^{y_{1}} \frac{\partial u}{\partial s}(x, s)$, so that $\psi^{\prime}(x)=$ $\int_{y_{2}}^{y_{1}} \frac{\partial^{2} u}{\partial x \partial s}(x, s) d s$ satisfies $\left|\psi^{\prime}(x)\right| \geq\left|y_{1}-y_{2}\right|$ on $[0,1]$ by the hypothesis $u \in \mathcal{U}_{1,1}$. So, by the one-dimensional version of the problem under consideration,

$$
|\{x:|\psi(x)| \leq 2 \alpha\}| \leq 4 \alpha /\left|y_{1}-y_{2}\right| .
$$

For a further discussion of Lemma 3.6, see Section 6 below.

Proof of Theorem 3.4. The proof is by the method of $T^{*} T$, although we phrase it without this terminology in view of later proofs. Assuming as we may that $f \geq 0$,

$$
\begin{aligned}
\left\|S_{\alpha} f\right\|_{2}^{2} & =\int\left|S_{\alpha} f(x)\right|^{2} d x \\
& =\int\left(\int \chi_{E_{\alpha}}(x, y) f(y) d y\right)^{2} d x \\
& =\iiint \chi_{E_{\alpha}}\left(x, y_{1}\right) f\left(y_{1}\right) \chi_{E_{\alpha}}\left(x, y_{2}\right) f\left(y_{2}\right) d y_{1} d y_{2} d x \\
& =\iint\left|E\left(y_{1}\right) \cap E\left(y_{2}\right)\right| f\left(y_{1}\right) f\left(y_{2}\right) d y_{1} d y_{2} \\
& \leq \iiint_{\left|y_{1}-y_{2}\right| \leq 4 \alpha} f\left(y_{1}\right) f\left(y_{2}\right) d y_{1} d y_{2}+4 \alpha \iint_{\left|y_{1}-y_{2}\right| \geq 4 \alpha} \frac{f\left(y_{1}\right) f\left(y_{2}\right) d y_{1} d y_{2}}{\left|y_{1}-y_{2}\right|} \\
& \leq 4 \alpha\|f\|_{2}^{2}+4 \alpha \log (1 / 4 \alpha)\|f\|_{2}^{2} \\
& \leq C \alpha \log (1 / \alpha)\|f\|_{2}^{2},
\end{aligned}
$$

as required.

The only property of the sublevel set $E=\{(x, y):|u(x, y)|<\alpha\}$ used in the proof of Corollary 3.5 was the inequality (3.4); the following example shows that the apparently natural upper bound of $C \alpha^{1 / 2}$ for $|E|$ does not follow from that property alone. Write $\mathcal{E}_{x}=\{y:(x, y) \in E\}$.

Proposition 3.7. For any $A<\infty$ there exist a parameter $\alpha$ and a set $E \subset[0,1]^{2}$ such that

$$
\left|\mathcal{E}_{x} \cap \mathcal{E}_{x^{\prime}}\right| \leq \alpha /\left|x-x^{\prime}\right| \quad \text { for every } x \neq x^{\prime} \in[0,1],
$$

but $|E|>A \alpha^{1 / 2}$. 
Proof. Let $\delta>0$ be a small parameter. Let $K \subset \mathbb{R}^{2}$ be a $\delta$-Kakeya/Besicovitch set, as constructed in [F], pp. 97-98. Define $\sigma=\sigma(\delta)$ to be the Lebesgue measure $|K|$ of $K$. Then $\sigma=O\left(\log \log \left(\delta^{-1}\right) / \log \left(\delta^{-1}\right)\right)$ tends $^{3}$ to zero as $\delta \rightarrow 0$. Moreover, to each $x \in[0,1]$ is associated a measurable set $T_{x} \subset K$, with $\left|T_{x}\right| \sim \delta$ and

$$
\left|T_{x} \cap T_{x^{\prime}}\right| \leq B \delta^{2} /\left|x-x^{\prime}\right|
$$

for some constant $B$. Finally $\left\{(x, y) \in[0,1] \times \mathbb{R}^{2}: y \in T_{x}\right\}$ is Borel measurable.

Indeed, the set $K$ constructed in $[\mathrm{F}]$ is a union of $\sim \delta^{-1}$ overlapping triangles. We interpret the parameter $x$ as an angle in a certain fixed range, and to each such parameter associate a triangle containing a line segment of unit length, pointing in the associated direction.

Fix a measurable map $\Phi:[0,1] \mapsto K$ satisfying $|\Phi(S)|=\sigma|S|$ for every measurable subset $S$ of $[0,1]$. Define

$$
E=\left\{(x, y) \in Q: \Phi(y) \in T_{x}\right\} .
$$

Define $\alpha=B \delta^{2} / \sigma$.

Now for each $x \in[0,1],\left|\mathcal{E}_{x}\right|=\sigma^{-1}\left|T_{x}\right| \sim \delta / \sigma$. Hence

$$
|E| \sim \delta / \sigma \sim \sigma^{-1 / 2} \alpha^{1 / 2} \gg \alpha^{1 / 2} .
$$

Moreover

$$
\left|\mathcal{E}_{x} \cap \mathcal{E}_{x^{\prime}}\right|=\sigma^{-1}\left|T_{x} \cap T_{x^{\prime}}\right| \leq B \delta^{2} \sigma^{-1} /\left|x-x^{\prime}\right|=\alpha /\left|x-x^{\prime}\right| .
$$

Thus $E$ satisfies (3.5), and $\alpha^{-1 / 2}|E| \sim \sigma^{-1 / 2}$ tends to infinity as $\delta \rightarrow 0$.

Observe that the counterexample agrees with the bound $C \alpha^{1 / 2} \log (1 / \alpha)^{1 / 2}$ up to a relatively tame factor ${ }^{4}$ of magnitude $\sqrt{\log \log \left(\alpha^{-1}\right)}$.

We have presented Theorem 3.4 separately from the general two-dimensional result because of its simplicity, but it does illustrate the method underlying the general result. For the general case we can assume, by symmetry, that $1 \leq j \leq k \leq$ $d-1$ when considering the class $\tilde{\mathcal{U}}_{j, k}$. We first state a generalisation of Lemma 3.6.

Lemma 3.8. For each $j, k \geq 1$ there exists an absolute constant $C_{j, k}$ such that for every $u \in \mathcal{U}_{j, k}$ and every $0<\alpha<1 / 2$, the sets $E(y)=\{x \in[0,1]:|u(x, y)| \leq \alpha\}$ satisfy

$$
\left|E\left(y_{0}\right) \cap \cdots \cap E\left(y_{k}\right)\right| \leq C_{j, k} \alpha^{1 / j} \sum_{m=0}^{k} \prod_{\ell: \ell \neq m}\left|y_{\ell}-y_{m}\right|^{-1 / j} .
$$

Proof. For $y_{0}, \ldots, y_{k}$ fixed, let

$$
\psi(x)=\sum_{m=0}^{k} \pm k ! \prod_{\ell: \ell \neq m}\left|y_{\ell}-y_{m}\right|^{-1} u\left(x, y_{m}\right),
$$

where the \pm signs are as in Lemma 2.3. Suppose $x \in E\left(y_{0}\right) \cap \cdots \cap E\left(y_{k}\right)$. Then since $\left|u\left(x, y_{m}\right)\right| \leq \alpha$ for each $0 \leq m \leq k$ we have

$$
|\psi(x)| \leq \alpha k ! \sum_{m=0}^{k} \prod_{\ell: \ell \neq m}\left|y_{\ell}-y_{m}\right|^{-1} .
$$

\footnotetext{
${ }^{3}$ This value of $\sigma$ is obtained by taking $\delta^{-1}=2^{k}$ and choosing $1-\alpha=\log (k) / 2 k$ in the construction in $[\mathrm{F}]$.

${ }^{4}$ We believe that even this discrepancy might be eliminated by means of a recent result of U. Keich, but have not yet been able to verify this.
} 
Now by Lemma 2.3, $\left(\frac{\partial}{\partial x}\right)^{j} \psi(x)$ is equal for any $x$ to $\frac{\partial^{k}}{\partial y^{k}} \frac{\partial^{j}}{\partial x^{j}} u(x, \zeta)$ for some $\zeta$ (depending on $x$ ) in the convex hull of $\left\{y_{0}, \ldots, y_{k}\right\}$, and so $\left(\frac{\partial}{\partial x}\right)^{j} \psi(x) \geq 1$ on $[0,1]$ since $u \in \mathcal{U}_{j, k}$.

Therefore, by the one-dimensional version of the problem,

$$
\begin{aligned}
\mid\{x:|\psi(x)| & \left.\leq \alpha k ! \sum_{m=0}^{k} \prod_{\ell: \ell \neq m}\left|y_{\ell}-y_{m}\right|^{-1}\right\} \mid \\
& \leq C_{j} \alpha^{1 / j}(k !)^{1 / j}\left\{\sum_{m=0}^{k} \prod_{\ell: \ell \neq m}\left|y_{\ell}-y_{m}\right|^{-1}\right\}^{1 / j} \\
& \leq C_{j, k} \alpha^{1 / j} \sum_{m=0}^{k} \prod_{\ell: \ell \neq m}\left|y_{\ell}-y_{m}\right|^{-1 / j}
\end{aligned}
$$

For an alternative approach to Lemmas 3.6 and 3.8 see Lemma 6.2.

Theorem 3.9. Let $1 \leq j \leq k$, and let $p=\frac{j(k+1)}{j(k+1)-k}, q=k+1$. Then there is an absolute constant $C_{j, k}$ so that for any integrable function $u$ satisfying $\partial^{j+k} u / \partial x^{j} \partial y^{k} \geq$ 1 on $Q$ (that is, for every $u \in \tilde{\mathcal{U}}_{j, k}$ ), for $0<\alpha<1$,

$$
\left\|S_{\alpha}\right\|_{L^{p} \rightarrow L^{q}} \leq C_{j, k} \begin{cases}\alpha^{1 / j(k+1)}, & j>1, \\ \alpha^{1 /(k+1)}\left(\log \alpha^{-1}\right)^{k /(k+1)}, & j=1 .\end{cases}
$$

Corollary 3.10. There is an absolute constant $C_{j, k}$ so that under the same hypothesis on $u$,

$$
|\{(x, y) \in Q:|u(x, y)| \leq \alpha\}| \leq C_{j, k} \begin{cases}\alpha^{\frac{1}{j(k+1)}}, & j>1 \\ \alpha^{\frac{1}{k+1}}\left(\log \alpha^{-1}\right)^{\frac{k}{k+1}}, & j=1 .\end{cases}
$$

Proof of Theorem 3.9.

$$
\begin{aligned}
\int\left(S_{\alpha} f\right)^{k+1} d x & =\int\left(\int \chi_{E_{\alpha}}(x, y) f(y) d y\right)^{k+1} d x \\
& =\int \cdots \int \prod_{m=1}^{k+1} \chi_{E_{\alpha}}\left(x, y_{m}\right) d x f\left(y_{1}\right) \cdots f\left(y_{k+1}\right) d y_{1} \cdots d y_{k+1} \\
& =\int \cdots \int\left|E\left(y_{1}\right) \cap \cdots \cap E\left(y_{k+1}\right)\right| f\left(y_{1}\right) \cdots f\left(y_{k+1}\right) d y_{1} \cdots d y_{k+1} .
\end{aligned}
$$

Suppose now that $j>1$; we use Lemma 3.8 and symmetry to dominate this expression by

$$
\begin{aligned}
& C \alpha^{1 / j} \int \cdots \int \quad\left|y_{1}-y_{2}\right|^{-1 / j}\left|y_{1}-y_{3}\right|^{-1 / j} \cdots\left|y_{1}-y_{k+1}\right|^{-1 / j} \\
& \cdot f\left(y_{2}\right) f\left(y_{3}\right) \cdots f\left(y_{k+1}\right) d y_{2} \cdots d y_{k+1} f\left(y_{1}\right) d y_{1} \\
& =C \alpha^{1 / j} \int\left\{I_{\beta} f\left(y_{1}\right)\right\}^{k} f\left(y_{1}\right) d y_{1}
\end{aligned}
$$


(where $I_{\beta}$ is fractional integration of order $\beta=1-\frac{1}{j}$ ) and hence by $\alpha^{1 / j}\left\|I_{\beta} f\right\|_{k p^{\prime}}^{k}\|f\|_{p}$. This in turn is dominated by $\alpha^{1 / j}\|f\|_{p}^{k+1}$ precisely when $\frac{1}{k p^{\prime}}=\frac{1}{p}-1+\frac{1}{j}$; that is, when $p=j(k+1) /(j(k+1)-k)$.

When $j=1$ we instead use multilinear interpolation with one copy of $f$ in $L^{1}$ and all the others in $L^{\infty}$. We use the estimate

$$
\left|E\left(y_{1}\right) \cap \cdots \cap E\left(y_{k+1}\right)\right| \leq C_{j, k} \min \left(1, \alpha \sum_{m=1}^{k+1} \prod_{\ell: \ell \neq m}\left|y_{\ell}-y_{m}\right|^{-1}\right)
$$

together with the fact that for each $s \geq 1$, the $L^{1}$ norm of $\min \left(1, \mu / t \log ^{s}(t / \mu)\right)$ as a function of $t \in[0,1]$ is $O\left(\mu \log ^{(s+1)}\left(\mu^{-1}\right)\right)$. Hence one incurs an extra factor of $\log (1 / \alpha)^{k}$ in this case.

Remark. Note that $\frac{1}{k+1} \leq \frac{j}{j+k}=\frac{j}{d}$ (with equality only when $j=1$ ), that $\left(\frac{1}{p}, \frac{1}{q}\right)=$ $\left(\frac{j(k+1)-k}{j(k+1)}, \frac{1}{k+1}\right)$ lies on the line $\frac{1}{j q}=\frac{1}{k p^{\prime}}$, and that the exponent $1 /(k+1) j$ equals $1 / j q=1 / k p^{\prime}$. Thus according to Corollary 3.2, the estimate of Theorem 3.9 is sharp (modulo a logarithmic factor in the case $j=1$ ) for the stated exponents $p, q$ for all $j, k$; however, we obtain the endpoint exponent pair $\left(p^{-1}, q^{-1}\right)=(j / d, j / d)$ only when $j=1$. Consequently we obtain a poor lower bound in Corollary 3.10 for large $j$; we know of no reason to believe that estimate to be sharp.

3.2. The two-dimensional case with extra hypotheses. We now discuss how sharp operator bounds for $S_{\alpha}$ may be obtained under some mild conditions of a qualitative nature on $u$. Before doing so we point out that according to Lemma 3.8, it suffices to obtain the desired operator bounds when the sublevel sets $E(y)$ satisfy

$$
\left|E\left(y_{1}\right) \cap \cdots \cap E\left(y_{k+1}\right)\right| \leq C_{j, k} \alpha^{1 / j} \sum_{m=1}^{k+1} \prod_{\ell: \ell \neq m}\left|y_{\ell}-y_{m}\right|^{-1 / j}
$$

for all $y_{1}, \ldots, y_{k+1}$. Note that if the $y_{m}$ are $\mu$-separated (i.e. $m \neq \ell \Rightarrow\left|y_{m}-y_{\ell}\right| \geq$ $\mu)$, (3.6) implies

$$
\left|E\left(y_{1}\right) \cap \cdots \cap E\left(y_{k+1}\right)\right| \leq C_{j, k} \alpha^{1 / j} / \mu^{k / j} .
$$

Let $L^{p, q}$ denote the usual Lorentz space.

In what follows we let $\mathcal{E}(x)=\{y:|u(x, y)| \leq \alpha\}$; the script $\mathcal{E}(x)$ denotes a set of parameters $y$ depending on $x$, while roman $E(y)$ denotes a set of parameters $x$ depending on $y$.

Proposition 3.11. Let $j, k \geq 1$ and set $d=j+k$. Then for each $A \geq 1$ there exists $C<\infty$ such that for any $u \in \mathcal{U}_{j, k}$ for which each set $\mathcal{E}(x)$ is a union of at most $A$ intervals,

$$
\left\|S_{\alpha} f\right\|_{L^{\frac{d}{j}, \infty}} \leq C \alpha^{1 / d}\|f\|_{\frac{d}{j}, 1} .
$$

Proof. It is no loss of generality to assume that each section $\mathcal{E}(x)=\left\{y:(x, y) \in E_{\alpha}\right\}$ is a single interval, for by hypothesis the sublevel set $E_{\alpha}$ may be decomposed as a union of at most $A$ sets, for each of which every section is an interval. These sets may not be sublevel sets, but their sections inherit (3.6), which is the only property of $E_{\alpha}$ to be used in the proof.

We may also assume that $f \equiv \chi_{F}$, and then that $\lambda \leq 1$. We want to show that

$$
|\{x:|\mathcal{E}(x) \cap F|>\lambda\}| \leq C \alpha^{1 / j}|F| \lambda^{-d / j} .
$$


We break $[0,1]$ up into equally spaced intervals $I_{\sigma}$, with spacing equal to the nearest integer to the reciprocal of $\alpha^{1 / j} / \lambda^{k / j}$. It is then enough to show that

$$
\alpha^{\frac{1}{j}} \lambda^{-\frac{k}{j}} \#\left\{\sigma: I_{\sigma} \text { contains an } x_{\sigma} \text { such that }\left|\mathcal{E}\left(x_{\sigma}\right) \cap F\right|>\lambda\right\} \leq C \alpha^{\frac{1}{j}}|F| / \lambda^{\frac{d}{j}} .
$$

Hence it suffices to show that

$$
\#\left\{\sigma: I_{\sigma} \text { contains an } x_{\sigma} \text { such that }\left|\mathcal{E}\left(x_{\sigma}\right) \cap F\right|>\lambda\right\} \leq C|F| \lambda^{-1} .
$$

By only considering every $M^{\prime}$ th interval, we may assume (at the expense of a factor $M$ ) that the $x_{\sigma}$ are $M \alpha^{\frac{1}{j}} / \lambda^{\frac{k}{j}}$-separated. By applying the remark before the statement of Proposition 3.11 with the roles of $x$ and $y$ interchanged, we see that by $(3.7)$

$$
\left|\mathcal{E}\left(x_{\sigma_{1}}\right) \cap \cdots \cap \mathcal{E}\left(x_{\sigma_{j+1}}\right)\right| \leq C \alpha^{\frac{1}{k}} /\left[M \alpha^{\frac{1}{j}} / \lambda^{\frac{k}{j}}\right]^{\frac{j}{k}}=C \lambda M^{-\frac{j}{k}} .
$$

Now (3.8) with $M$ sufficiently large, together with the fact that each $\left|\mathcal{E}\left(x_{\sigma}\right)\right|>\lambda$, implies that the intervals $\mathcal{E}\left(x_{\sigma}\right)$ have bounded overlap, i.e. no point in $[0,1]$ belongs to more than $N=N(j, k)$ of them. Thus

$$
\begin{aligned}
|F| & \geq \int \chi_{F} \chi_{\sigma} \mathcal{E}\left(x_{\sigma}\right) \geq \frac{1}{N} \int \chi_{F} \sum_{\sigma} \chi_{\mathcal{E}\left(x_{\sigma}\right)}=\frac{1}{N} \sum_{\sigma}\left|F \cap \mathcal{E}\left(x_{\sigma}\right)\right| \\
& \geq \frac{1}{N} \lambda \#\left\{\sigma: I_{\sigma} \text { contains an } x_{\sigma} \text { with }\left|\mathcal{E}\left(x_{\sigma}\right) \cap F\right|>\lambda\right\}
\end{aligned}
$$

which is what we needed to finish the proof.

Corollary 3.12. Under the same hypotheses as Proposition 3.11

$$
|E|=|\{(x, y):|u(x, y)| \leq \alpha\}| \leq C \alpha^{\frac{1}{d}}
$$

where $C$ depends only on $j, k$ and $A$.

Proof.

$$
|E|=\left\|S_{\alpha} 1\right\|_{1} \leq\left\|S_{\alpha} 1\right\|_{d / j, \infty}\|1\|_{d / k, 1} \leq C \alpha^{\frac{1}{d}}\|1\|_{d / j, 1}\|1\|_{d / k, 1}=C \alpha^{\frac{1}{d}} .
$$

With some extra effort, one may improve the restricted weak-type conclusion of Proposition 3.11 to the expected strong-type conclusion.

Theorem 3.13. Suppose that $E \subseteq[0,1]^{2}$. Let $\mathcal{E}(x)=\{y:(x, y) \in E\}$. Suppose that each $\mathcal{E}(x)$ is an interval. Suppose furthermore that for certain $j, k \in \mathbb{N}$,

$$
\left|\mathcal{E}\left(x_{0}\right) \cap \mathcal{E}\left(x_{1}\right) \cap \cdots \cap \mathcal{E}\left(x_{j}\right)\right| \leq C \alpha^{1 / k} \sum_{s=0}^{j} \prod_{r: r \neq s}\left|x_{r}-x_{s}\right|^{-1 / k}
$$

for all $x_{0}, \ldots, x_{j} \in[0,1]$. Then the operator $S f(x)=\int \chi_{E}(x, y) f(y) d y$ satisfies

$$
\|S f\|_{\frac{j+k}{j}} \leq C \alpha^{\frac{1}{j+k}}\|f\|_{\frac{j+k}{j}} .
$$

Proof. In the first three steps of the proof, we will assume that each interval $\mathcal{E}(x)$ is dyadic. 
Step 1: $\|S(1)\|_{\frac{j+k}{j}} \leq C \alpha^{\frac{1}{j+k}}$.

To establish this, it suffices to show that

$$
\int_{\{x: \mathcal{E}(x) \subseteq I\}}|\mathcal{E}(x)|^{\frac{j+k}{j}} d x \leq C \alpha^{\frac{1}{j}}|I|
$$

whenever $I$ is a dyadic interval which occurs as some $\mathcal{E}\left(x_{I}\right)$. For, given (3.10), one can choose $I$ to be a maximal dyadic interval occurring amongst $\{\mathcal{E}(x): x \in[0,1]\}$, and then sum over the (disjoint) $I$ occurring.

If $j=1$ we can use the fact that if $x_{I}$ is such that $\mathcal{E}\left(x_{I}\right)=I$, then $|\mathcal{E}(x)| \leq$ $C \min \left(\alpha^{1 / k}\left|x-x_{I}\right|^{-1 / k},|I|\right)$ whenever $\mathcal{E} \subset I$, by (3.9). Integrating this raised to a suitable power gives $(3.10) .^{5}$

Assume now that $j \geq 2$. For $I$ fixed we estimate the left-hand side of (3.10) as follows. It suffices to show, for each $p=0,1,2, \ldots$,

$$
\int_{\left\{x: \mathcal{E}(x) \subseteq I,|\mathcal{E}(x)|=|I| \cdot 2^{-p}\right\}}|\mathcal{E}(x)|^{\frac{j+k}{j}} d x \leq C \alpha^{\frac{1}{j}}|I| 2^{-\frac{p}{j}} .
$$

For each such $p$ we decompose $I$ as a disjoint union of dyadic intervals $I_{\lambda}$, with each $\left|I_{\lambda}\right|=|I| \cdot 2^{-p}$. Let

$$
\begin{aligned}
S_{\lambda} & =\left\{x: \mathcal{E}(x)=I_{\lambda}\right\} \\
F_{0} & =\left\{\lambda:\left|S_{\lambda}\right| \leq 2^{-\frac{p}{j}} \alpha^{\frac{1}{j}}\left(2^{-p}|I|\right)^{-\frac{k}{j}}\right\}
\end{aligned}
$$

and, for $m \geq 1$,

$$
F_{m}=\left\{\lambda:\left|S_{\lambda}\right| \sim 2^{m} 2^{-\frac{p}{j}} \alpha^{\frac{1}{j}}\left(2^{-p}|I|\right)^{-\frac{k}{j}}\right\} .
$$

Then

$$
\begin{aligned}
\int_{\left\{x \in \mathcal{E}(x) \subseteq I,|\mathcal{E}(x)|=|I| 2^{-p}\right\}}|\mathcal{E}(x)|^{(j+k) / j} & \sim \sum_{m=0}^{\infty} \sum_{\lambda \in F_{m}} \int_{S_{\lambda}}|\mathcal{E}(x)|^{(j+k) / j} \\
& \sim \sum_{m=0}^{\infty} \sum_{\lambda \in F_{m}}\left|S_{\lambda}\right|\left(|I| 2^{-p}\right)^{(j+k) / j} \\
& \leq C \alpha^{\frac{1}{j}} 2^{-\frac{p}{j}}|I|\left(1+\sum_{m=1}^{\infty} \# F_{m} 2^{m} 2^{-p}\right) .
\end{aligned}
$$

Claim. For every $m \geq 1, \# F_{m} \leq C 2^{p-m j}$.

The Claim establishes (3.11) as we are assuming $j \geq 2$.

Proof of the Claim. Let $x_{0}$ satisfy $\mathcal{E}\left(x_{0}\right)=I$, and assume without loss of generality that each member of each $S_{\lambda}$ is greater than or equal to $x_{0}$. Pick a $\lambda_{0}$ such that $S_{\lambda_{0}}$ contains some member $\bar{x}$ such that

$$
\bar{x}-x_{0}>\frac{1}{2} D:=\frac{1}{2} \text { diameter }\left(\bigcup S_{\lambda}\right) .
$$

\footnotetext{
${ }^{5}$ We are indebted to Nets Katz for pointing out this argument; our original proof in the case $j=1$ was much more complicated.
} 
Now a suitable modification of Lemma 2.4 gives the existence of $x_{1}, x_{2}, \ldots, x_{j} \in S_{\lambda_{0}}$ such that for $0 \leq r, s \leq j$

$$
\prod_{\substack{s: s \neq r \\ s \in\{0, \ldots, j\}}}\left|x_{r}-x_{j}\right| \geq C\left|S_{\lambda_{0}}\right|^{j-1} D .
$$

Then, as $\mathcal{E}\left(x_{s}\right)=I_{\lambda_{0}}$ for $s=1, \ldots, j$, we have by $(3.9)$

$$
\begin{aligned}
2^{-p}|I| & =\left|\bigcap_{s=0}^{j} \mathcal{E}\left(x_{s}\right)\right| \leq \frac{C \alpha^{\frac{1}{k}}}{\min _{r} \prod_{s: s \neq r}\left|x_{r}-x_{s}\right|^{\frac{1}{k}}} \\
& \leq \frac{C \alpha^{\frac{1}{k}}}{\left\{\left|S_{\lambda_{0}}\right|^{j-1} D\right\}^{\frac{1}{k}}} \leq \frac{C \alpha^{\frac{1}{k}}}{\left|S_{\lambda_{0}}\right|^{\frac{j}{k}}\left(\# F_{m}\right)^{\frac{1}{k}}},
\end{aligned}
$$

since $D \geq \#\left(F_{m}\right)\left|S_{\lambda_{0}}\right|$. Therefore

$$
\# F_{m} \leq \frac{C \alpha}{\left|S_{\lambda_{0}}\right|^{j}\left(2^{-p}|I|\right)^{k}} \sim C 2^{-m j} 2^{p} .
$$

Step 1 is now complete.

Step 2: For a dyadic interval $J$,

$$
\left\|S \chi_{J}\right\|_{\frac{j+k}{j}} \leq C \alpha^{\frac{1}{j+k}}|J|^{\frac{j}{j+k}} .
$$

This follows directly from Step 1 , by replacing each interval $\mathcal{E}(x)$ by $\mathcal{E}(x) \cap J$. These smaller intervals still satisfy (3.9), which is all that was used in the proof of (3.10). Invoking (3.10) for each maximal dyadic interval $I \subset J$ in the resulting collection, and then summing over all such maximal intervals, yields the conclusion desired.

Step 3: $\|S f\|_{\frac{j+k}{j}} \leq C \alpha^{\frac{1}{j+k}}\|f\|_{\frac{j+k}{j}}$ for all $f$.

For this we merely observe that, $\mathcal{D}^{j}$ denoting the collection of all dyadic intervals in $[0,1]$ and $f_{I}$ denoting $|I|^{-1} \int_{I} f$,

$$
\begin{aligned}
\int|S f|^{\frac{j+k}{j}} & =\sum_{I \in \mathcal{D}} \int_{\{x: \mathcal{E}(x)=I\}}\left(\int_{I} f\right)^{\frac{j+k}{j}} \\
& =\sum_{I \in \mathcal{D}}\left(f_{I}\right)^{\frac{j+k}{j}}|I|^{\frac{j+k}{j}}|\{x: \mathcal{E}(x)=I\}| \\
& =\sum_{I \in \mathcal{D}}\left(f_{I}\right)^{\frac{j+k}{j}} \gamma_{I},
\end{aligned}
$$

where $\gamma_{I}$ is defined by the last identity. Now this quantity is bounded above by $\alpha^{\frac{1}{j}}\|f\|_{(j+k) / j}^{(j+k) / j}$ for general $f$ if and only if $\left\{\gamma_{I}\right\}$ satisfies the Carleson measure condition

$$
\sum_{I \subseteq J} \gamma_{I} \leq C \alpha^{\frac{1}{j}}|J| \quad \text { for all } J
$$

(See, for example, [S].) But

$$
\sum_{I \subseteq J} \gamma_{I} \leq \int\left|S \chi_{J}\right|^{\frac{j+k}{j}} \leq C \alpha^{\frac{1}{j}}|J|
$$

by Step 2. This concludes the proof, in the case where all intervals are dyadic. 
Step 4: The general case.

To each $x$ associate a dyadic interval $\tilde{I}(x) \subset \mathcal{E}(x)$, such that $|\tilde{I}(x)| \geq|\tilde{\mathcal{E}}(x)| / 4$. To $x$ associate also $I^{*}(x)$, defined to be the union of 9 adjacent dyadic intervals each of length $|\tilde{I}(x)|$, such that the midpoint of $\tilde{I}(x)$ is the midpoint of their union. Then $\mathcal{E}(x) \subset I^{*}(x)$.

The collection $\{\tilde{I}(x)\}$ certainly still satisfies the hypothesis (3.9), hence satisfies the conclusion of Step 2 above. It follows almost immediately that $\left\{I^{*}(x)\right\}$ likewise satisfies that conclusion. The proof of Step 3 established that the conclusion of Step 2 implies the desired operator estimate. Since the operator associated to the larger intervals $I^{*}(x)$ dominates our operator $S$, the proof is complete.

In the next corollary, by a single-signed distribution we mean one that is realvalued and is either nonnegative on every open set, or nonpositive on every open set.

Corollary 3.14. Let $u \in \tilde{\mathcal{U}}_{j, k}$ and suppose either that for some $N>k, \partial^{N} u / \partial y^{N}$ is single-signed, or that for some $N>j, \partial^{N} u / \partial x^{N}$ is single-signed. Then there exists a finite constant $C$ depending only on $j, k, N$ such that

$$
\left\|S_{\alpha} f\right\|_{\frac{j+k}{j}} \leq C \alpha^{1 /(j+k)}\|f\|_{\frac{j+k}{j}} \quad \text { for all } f, \alpha .
$$

Since the hypotheses are stable under convolution with nonnegative approximate identities, the corollary follows from Theorem 3.13 by regularisation.

3.3. The higher-dimensional case. When $n \geq 3$ our results are less complete. We will show that there always are nontrivial uniform $L^{p}-L^{q}$ operator bounds, and that under certain nonquantitative "convexity" conditions imposed on $u$, optimal estimates can be achieved for the sublevel set problem.

Theorem 3.15. For each $n \geq 1$ and each $\beta \in \mathbb{N}^{n}$ there exist $\varepsilon>0$ and $C<\infty$ such that for any real-valued, integrable function $u$ satisfying $D^{\beta} u \geq 1$ in the sense of distributions on $Q$, and for any $\alpha>0$,

$$
|\{x \in Q:|u(x)| \leq \alpha\}| \leq C \alpha^{\varepsilon} .
$$

This conclusion should be contrasted with a previously known result. If $u \in C^{|\beta|}$, then the conclusion holds with $\varepsilon=1 /|\beta|$, with a constant $C$ that depends on $u$. See for instance [S]. Our conclusion is uniform over a large class of $u$, but with a weaker exponent.

Theorem 3.16. For each $n=n^{\prime}+n^{\prime \prime} \geq 2$, each $1 \leq p, q \leq \infty$ such that $p \neq 1$ and $q \neq \infty$, and each $\beta$ there exist $\varepsilon=\varepsilon(n, \beta, p, q)>0$ and $C=C(\varepsilon, n, \beta, p, q)$ such that whenever $u$ is real-valued, integrable and $D^{\beta} u \geq 1$ in the sense of distributions on $Q$, for each $\alpha>0$ the operator

$$
S_{\alpha} f\left(x^{\prime}\right)=\int_{Q^{n^{\prime \prime}}} \chi_{\left\{\left|u\left(x^{\prime}, x^{\prime \prime}\right)\right| \leq \alpha\right\}} f\left(x^{\prime \prime}\right) d x^{\prime \prime}
$$

satisfies

$$
\left\|S_{\alpha} f\right\|_{L^{q}\left(Q^{n^{\prime}}\right)} \leq C \alpha^{\varepsilon}\|f\|_{L^{p}\left(Q^{n^{\prime \prime}}\right)} .
$$

Theorem 3.16 is an immediate consequence of Theorem 3.15 so we prove only the latter. Throughout its proof we suppose, without loss of generality, that $u \in C^{\infty}$, that $D^{\beta} u \geq 1$ on $Q=Q^{n-1} \times Q^{1}$, and that $\beta_{n} \neq 0$. 
Proof of Theorem 3.15. The proof is by induction on $n$. The case $n=2$ has already been dealt with as Corollary 3.10, and so we assume the case $n-1$ of Theorem 3.15. Without loss of generality we may assume that $u \in C^{\infty}$.

Let $\beta=\left(\beta^{\prime}, \beta_{n}\right) \in \mathbb{N}^{n-1} \times \mathbb{N}$ and write $\beta_{n}=k$. Let $E=\left\{x \in Q^{n}:|u(x)| \leq \alpha\right\}$ and $E(y)=\left\{x^{\prime}:\left(x^{\prime}, y\right) \in E\right\}$. We first claim that

$$
\left|E\left(y_{1}\right) \cap \cdots \cap E\left(y_{k+1}\right)\right|_{n-1} \leq C \alpha^{\varepsilon\left(n-1, \beta^{\prime}\right)} \sum_{m=1}^{k+1} \prod_{\ell: \ell \neq m}\left|y_{\ell}-y_{m}\right|^{-\varepsilon\left(n-1, \beta^{\prime}\right)}
$$

as in Lemma 3.8, where the left-hand side denotes the $n-1$ dimensional Hausdorff measure of the set indicated. Indeed, with an appropriate choice of the \pm signs, defining

$$
\psi\left(x^{\prime}\right)=\sum_{m=1}^{k+1} \pm k ! \prod_{\ell: \ell \neq m}\left|y_{\ell}-y_{m}\right|^{-1} u\left(x^{\prime}, y_{m}\right)
$$

we have

$$
D^{\beta^{\prime}} \psi\left(x^{\prime}\right)=\partial_{y}^{k} D^{\beta^{\prime}} u\left(x^{\prime}, \zeta\right)
$$

for some $\zeta$ in the convex hull of $\left\{y_{1}, \ldots, y_{k+1}\right\}$ by the higher-order mean-value theorem, Lemma 2.3. So $D^{\beta^{\prime}} \psi \geq 1$ on $Q^{n-1}$. Thus, by the inductive hypothesis,

$$
\left|\left\{x^{\prime}:\left|\psi\left(x^{\prime}\right)\right| \leq \gamma\right\}\right|_{n-1} \leq C \gamma^{\varepsilon\left(n-1, \beta^{\prime}\right)} .
$$

On the other hand, $x^{\prime} \in E\left(y_{1}\right) \cap \cdots \cap E\left(y_{k+1}\right)$ implies

$$
\left|\psi\left(x^{\prime}\right)\right| \leq C \alpha \sum_{m=1}^{k+1} \prod_{\ell: \ell \neq m}\left|y_{\ell}-y_{m}\right|^{-1}
$$

and so (3.13) follows. Now

$$
\begin{aligned}
|E| & =\int_{Q^{n-1}} \int_{Q^{1}} \chi_{E}\left(x^{\prime}, y\right) d y d x^{\prime} \\
& \leq\left\{\int_{Q^{n-1}}\left\{\int_{Q^{1}} \chi_{E}\left(x^{\prime}, y\right) d y\right\}^{k+1} d x^{\prime}\right\}^{\frac{1}{k+1}} \\
& =\left\{\int \cdots \int\left|E\left(y_{1}\right) \cap \cdots \cap E\left(y_{k+1}\right)\right|_{n-1} d y_{1} \ldots d y_{k+1}\right\}^{\frac{1}{k+1}} \\
& \leq C \alpha^{\varepsilon\left(n-1, \beta^{\prime}\right) /(k+1)}
\end{aligned}
$$

as required.

Notice that the proof shows that we may take $\varepsilon(n, \beta)=\varepsilon\left(n-1, \beta^{\prime}\right) /\left(\beta_{n}+1\right)$. As in Theorem 3.9 there is also an operator-theoretic $L^{p}-L^{q}$ estimate implicit in the above argument. For $n=3, \beta=(1,1,1)$ we obtain

$$
\left\|S_{\alpha} f\right\|_{L^{2}\left(Q^{2}\right)} \leq C \alpha^{\frac{1}{4}} \log (1 / \alpha)^{C}\|f\|_{L^{\frac{4}{3}}\left(Q^{1}\right)} .
$$

However, even here one uses induction on the measure estimate rather than the operator-norm estimate. 
Simple examples (as in the two-dimensional case) show that no better result is possible for $S_{\alpha}$ under the hypothesis $D_{x^{\prime}}^{\beta^{\prime}} D_{x^{\prime \prime}}^{\beta^{\prime \prime}} u \geq 1$ than

$$
\left\|S_{\alpha} f\right\|_{r} \leq C \alpha^{\frac{1}{\left|\beta^{\prime}\right|+\left|\beta^{\prime \prime}\right|}}\|f\|_{r}
$$

with $r=\left(\left|\beta^{\prime}\right|+\left|\beta^{\prime \prime}\right|\right) /\left|\beta^{\prime}\right|$.

Conversely, further "convexity" conditions on $u$ lead to the corresponding optimal sublevel set estimate:

Proposition 3.17. Suppose that $u$ is integrable, that $D^{\beta} u \geq 1$ in the sense of distributions on $Q$, and moreover that for some indices $N_{2}>\beta_{2}, N_{3}>\beta_{3}, \ldots, N_{n}>$ $\beta_{n}$, the partial derivatives $D^{\left(0,0, \ldots, N_{n}\right)} u, D^{\left(0,0, \ldots, 0, N_{n-1}, \beta_{n}\right)} u, \ldots, D^{\left(0, N_{2}, \beta_{3} \ldots, \beta_{n}\right)} u$ are all single-signed, as distributions. There there is a $C<\infty$ depending only on $\beta, N_{2}, \ldots, N_{n}$ such that for every $\alpha>0$,

$$
|\{x \in Q:|u(x)| \leq \alpha\}| \leq C \alpha^{1 /|\beta|} .
$$

Proof. By the usual approximation argument we may assume that $u \in C^{\infty}$. The proof proceeds by induction on $n$. The case $n=1$ is Proposition 2.1 and so we assume the statement of Proposition 3.17 to hold in the case $n-1$. If $E=$ $\{x \in Q:|u(x)| \leq \alpha\}$ we let $\gamma$ denote a quantity to be chosen below, and write $|E|=\int_{Q} \chi_{E}\left(x^{\prime}, x_{n}\right) d x=I+I I$, where $I$ is the integral over the set where $\left|\partial^{\beta_{n}} u(x) / \partial x_{n}^{\beta_{n}}\right| \geq \gamma$.

For $I I$ we use the inductive hypothesis applied to $D^{\beta^{\prime}}$ with $\left(\frac{\partial}{\partial x_{n}}\right)^{\beta_{n}} u$ in place of $u$ to estimate the inner integral by $C \gamma^{\frac{1}{\left|\beta^{\prime}\right|}}$. For $I$ we fix $x^{\prime}$ and estimate the inner integral by $C(\alpha / \gamma)^{\frac{1}{\beta_{n}}}$ using the single-signedness of $\left(\frac{\partial}{\partial x_{n}}\right)^{N_{n}} u$, which assures us that $[0,1]$ decomposes into at most boundedly many intervals on each of which $\partial^{\beta_{n}} u / \partial x_{n}^{\beta_{n}}$ is single-signed. Thus $|E| \leq C\left(\gamma^{\frac{1}{\left|\beta^{\prime}\right|}}+(\alpha / \gamma)^{\frac{1}{\beta_{n}}}\right)$ which, upon taking $\gamma=\alpha^{\left|\beta^{\prime}\right| /|\beta|}$, yields $|E| \leq C \alpha^{\frac{1}{|\beta|}}$ as desired.

An application of this proposition can be found in Section 7 .

Finally, we note that Proposition 3.17 can be strengthened slightly. Under its hypotheses, one obtains that the operator $S_{\alpha}$ maps $L^{\frac{|\beta|}{\beta_{1}}, 1}\left(Q^{n-1}\right)$ boundedly to $L^{\frac{|\beta|}{\beta_{1}}, \infty}\left(Q^{1}\right)$ with constant $O\left(\alpha^{1 /|\beta|}\right)$. One mimics the proof of Proposition 3.11 using in place of (3.8) the estimate

$$
\left|\mathcal{E}\left(x_{1}\right) \cap \cdots \cap \mathcal{E}\left(x_{\beta_{1}+1}\right)\right| \leq C \alpha^{1 / \beta_{1}} \sum_{m=1}^{\beta_{1}+1} \prod_{\ell: \ell \neq m}\left|x_{\ell}-x_{m}\right|^{-1 / \beta_{1}}
$$

which is established, using the convexity assumption and Proposition 3.17, implicitly in the proof of Theorem 3.15. The details are left to the reader. One may similarly expect to improve restricted weak type $|\beta| / \beta_{1}$ to strong type $|\beta| / \beta_{1}$ along the lines of Theorem 3.13. We do not pursue this point here.

\section{Oscillatory integral operators}

4.1. The two-dimensional case. Let, for $|\lambda|>>1$,

$$
T_{\lambda} f(x)=\int_{[0,1]} e^{i \lambda u(x, y)} f(y) d y \quad \text { and } \quad I(\lambda)=\int_{[0,1]^{2}} e^{i \lambda u(x, y)} d x d y
$$


where $u \in \tilde{\mathcal{U}}_{j, k}$ and $j, k>0$; that is, $u: Q \rightarrow \mathbb{R}$ is integrable and satisfies $\partial^{j+k} u / \partial x^{j} \partial y^{k} \geq 1$ on $Q=[0,1]^{2}$, in the distribution sense. We could just as well define $T_{\lambda}$ and $I(\lambda)$ incorporating smooth bump functions rather than the 'rough' cut-offs $\chi_{[0,1]}$ or $\chi_{Q}$, and our conclusions would be unchanged; either formulation of the results implies the other.

In this subsection we investigate the behaviour of $I(\lambda)$ and $\left\|T_{\lambda}\right\|_{L^{p} \rightarrow L^{q}}$ for large $|\lambda|, 1 \leq p, q \leq \infty$. As always, we are concerned only with estimates which are uniform over the class $\tilde{\mathcal{U}}_{j, k}$, or equivalently over the subclass $\mathcal{U}_{j, k}$ of smooth functions; for nonuniform results, see for example [S], p.416. If $j$ or $k$ is zero, then $I(\lambda)$ is essentially one-dimensional, at least from our point of view, and is governed by the classical van der Corput lemma; in contrast, there are no decay estimates for $\left\|T_{\lambda}\right\|$, as may be seen by considering functions $u$ of the appropriate single variable $x$ or $y$.

Proposition 4.1. If $j, k \geq 1$ and $\alpha>0$, then the statement

$$
|I(\lambda)| \leq C|\lambda|^{-\alpha} \text { for all } u \in \mathcal{U}_{j, k},
$$

with $C$ independent of $u$, is equivalent to

$$
\left\|T_{\lambda}\right\|_{L^{\infty} \rightarrow L^{1}} \leq C|\lambda|^{-\alpha} \text { for all } u \in \mathcal{U}_{j, k} .
$$

Proof. If $\left\|T_{\lambda} f\right\|_{1} \leq C|\lambda|^{-\alpha}\|f\|_{\infty}$, then $\left|\int T_{\lambda} f(x) g(x) d x\right| \leq C \lambda^{-\alpha}\|f\|_{\infty}\|g\|_{\infty}$, and taking $f=g=1$ gives $|I(\lambda)| \leq C|\lambda|^{-\alpha}$. Conversely, suppose $|I(\lambda)| \leq C|\lambda|^{-\alpha}$ for all $u \in \mathcal{U}_{j, k}$. Then for arbitrary $\phi, \psi \in C^{\infty}$, and $u \in \mathcal{U}_{j, k}$

$$
\left|\int e^{i \lambda u(x, y)} e^{i \phi(x)} e^{i \psi(y)} d x d y\right| \leq C|\lambda|^{-\alpha}
$$

since $u(x, y)+\lambda^{-1} \phi(x)+\lambda^{-1} \psi(y) \in \mathcal{U}_{j, k}$ too when $j, k \geq 1$. Now let $A$ and $B$ be arbitrary measurable subsets of $[0,1]$ and choose sequences of smooth functions $\phi_{n}$ and $\psi_{n}$ so that

$$
\phi_{n}(x) \rightarrow\left\{\begin{array} { l l } 
{ 0 , } & { x \in A , } \\
{ \pi , } & { x \in A ^ { C } , }
\end{array} \quad \text { and } \quad \psi _ { n } ( y ) \rightarrow \left\{\begin{array}{ll}
0, & y \in B \\
\pi, & y \in B^{C} .
\end{array}\right.\right.
$$

By the dominated convergence theorem,

$$
\begin{aligned}
\int e^{i \lambda u(x, y)} e^{i \phi_{n}(x)} e^{i \psi_{n}(y)} d x d y \\
\rightarrow \int e^{i \lambda(x, y)}\left[\chi_{A}-\chi_{A^{C}}\right](x)\left[\chi_{B}-\chi_{B^{C}}\right](y) d x d y
\end{aligned}
$$

as $n \rightarrow \infty$. Hence the latter integral is $O\left(|\lambda|^{-\alpha}\right)$ uniformly in $A, B$ and $\lambda$. Combining this with the special cases where $A$ and/or $B$ is empty one immediately deduces that

$$
\left|\int e^{i \lambda u(x, y)} \chi_{A}(x) \chi_{B}(y) d x d y\right| \leq C|\lambda|^{-\alpha}
$$

for arbitrary measurable $A$ and $B$. Hence,

$$
\left|\int e^{i \lambda u(x, y)}\left(\sum_{\ell} \alpha_{\ell} \chi_{A_{\ell}}(x)\right)\left(\sum_{m} \beta_{m} \chi_{B_{m}}(y)\right) d x d y\right| \leq C|\lambda|^{-\alpha} \sum_{\ell}\left|\alpha_{\ell}\right| \sum_{m}\left|\beta_{m}\right|
$$


for arbitrary measurable $\left\{A_{\ell}\right\},\left\{B_{m}\right\}$. If now $f, g \in L^{\infty}[0,1]$, we can write $f$ as $\sum_{\ell} \alpha_{\ell} \chi_{A_{\ell}}$ with $\sum\left|\alpha_{\ell}\right| \leq\|f\|_{\infty}$ and similarly for $g$. Thus

$$
\left|\int e^{i \lambda u(x, y)} f(x) g(y) d x d y\right| \leq C|\lambda|^{-\alpha}\|f\|_{\infty}\|g\|_{\infty}
$$

and hence $\left\|T_{\lambda}\right\|_{L^{\infty} \rightarrow L^{1}} \leq C|\lambda|^{-\alpha}$.

Remark. Let $1 \leq p, q \leq \infty(p \neq 1, q \neq \infty)$. Then, by interpolation, the statements of Proposition 4.1 are also equivalent to $\left\|T_{\lambda}\right\|_{L^{p} \rightarrow L^{q}} \leq C|\lambda|^{-\alpha(p, q)}$.

Our first result is analogous to the failure of the classical van der Corput estimate under the hypothesis $\psi^{\prime} \geq 1$ but without any convexity assumption.

Theorem 4.2. There is no $\alpha>0$ such that for some absolute $C$, for all $u \in \mathcal{U}_{1,1}$, $|I(\lambda)| \leq C|\lambda|^{-\alpha}$. There are no $1 \leq p, q \leq \infty$ and no $\alpha>0$ for which one has $\left\|T_{\lambda}\right\|_{L^{p} \rightarrow L^{q}}=O\left(|\lambda|^{-\alpha}\right)$ uniformly in $u \in \mathcal{U}_{1,1}$.

By Proposition 4.1 it is enough to prove the first statement of Theorem 4.2. This we achieve with the help of some lemmas.

Lemma 4.3. For any $\varepsilon>0$, there exists $u \in \mathcal{U}_{1,1}$ such that $\operatorname{dist}(u(x, y), \mathbb{Z})<\varepsilon$ except for $(x, y)$ in a set $F_{\varepsilon}$ of measure at most $\varepsilon$.

Lemma 4.4. For each $M>>1$ and each $\varepsilon>0$ there is a smooth $g:[0,1] \rightarrow \mathbb{R}$ such that $g^{\prime}(y) \geq M$ on $[0,1]$ and $\operatorname{dist}(g(y), \mathbb{Z})<\varepsilon$ except for an exceptional set of $y$ of measure $O(\varepsilon)$ consisting of at most $C M / \varepsilon$ intervals each of length $\varepsilon^{2} / M$.

Proof of Lemma 4.4. Merely let $g(0)=0$ and make $g$ piecewise linear with slopes alternately $M / \varepsilon^{2}$ and $M$ on intervals of length $\approx \varepsilon^{2} / M$ and $\varepsilon / M$ respectively. Then $g$ can be made to be within $\varepsilon$ of $\mathbb{Z}$ except on the union of the intervals of length $\varepsilon^{2} / M$, of which one needs $\approx M / \varepsilon$ to construct $g$ on all of $[0,1]$. Finally one can convolve with an approximate identity to produce a smooth $g$.

Proof of Lemma 4.3. We first define inductively some auxiliary functions on $[0,1]$. Let $f_{0}=0$, and, given $f_{0}, f_{1}, \ldots, f_{j}$ each with the property that $\operatorname{dist}\left(f_{j}(y), \mathbb{Z}\right)<\varepsilon$ except on a set of measure $\varepsilon$, one uses Lemma 4.4 to define $f_{j+1}$ with the same property and such that

$$
\inf _{0 \leq y \leq 1} f_{j+1}^{\prime}(y) \geq \sup _{0 \leq y \leq 1} f_{j}^{\prime}(y)+\varepsilon .
$$

We now define $f(x, y)$ on $Q=[0,1]^{2}$ as follows. For $0 \leq j \leq \frac{1}{\varepsilon}(\in \mathbb{N})$, let $f(j \varepsilon, y)=$ $f_{j}(y)$. For $j \varepsilon \leq x \leq(j+1) \varepsilon-\varepsilon^{2}$, one sets $f(x, y)=f_{j}(y)+(x-j \varepsilon) y$. For $(j+1) \varepsilon-\varepsilon^{2} \leq x \leq(j+1) \varepsilon$, one interpolates linearly (for each fixed $y$ ) between the values of $f\left((j+1) \varepsilon-\varepsilon^{2}, y\right)$ and $f((j+1) \varepsilon, y)$. That is,

$$
f(x, y)=\left[\frac{(j+1) \varepsilon-x}{\varepsilon^{2}}\right] f\left((j+1) \varepsilon-\varepsilon^{2}, y\right)+\left[\frac{x-(j+1) \varepsilon+\varepsilon^{2}}{\varepsilon^{2}}\right] f((j+1) \varepsilon, y)
$$

when $(j+1) \varepsilon-\varepsilon^{2} \leq x \leq(j+1) \varepsilon$.

Now, except on the lines $x=j \varepsilon$ and $x=j \varepsilon-\varepsilon^{2}$,

$$
\frac{\partial^{2} f}{\partial x \partial y}= \begin{cases}1, & j \varepsilon<x<(j+1) \varepsilon-\varepsilon^{2}, \\ \frac{1}{\varepsilon^{2}}\left[f_{j+1}^{\prime}(y)-f_{j}^{\prime}(y)-\varepsilon+\varepsilon^{2}\right], & (j+1) \varepsilon-\varepsilon^{2}<x<(j+1) \varepsilon,\end{cases}
$$

the value of which is at least 1 by the construction of the $f_{j}$ 's. 
For $x$ belonging to one of the strips $j \varepsilon<x<(j+1) \varepsilon-\varepsilon^{2}$, we have $\left|f(x, y)-f_{j}(y)\right|$ $=|(x-j \varepsilon) y| \leq \varepsilon$, so that $f(x, y)$ is within $2 \varepsilon$ of $\mathbb{Z}$ except on a union of horizontal strips of total two-dimensional measure $\varepsilon \times \varepsilon=\varepsilon^{2}$, for each fixed $j$. As there are $\frac{1}{\varepsilon}$ such $j$ 's this gives an exceptional set of measure $\varepsilon$ altogether.

The remaining strips $(j+1) \varepsilon-\varepsilon^{2}<x<(j+1) \varepsilon$ each have measure $\varepsilon^{2}$ and there are $\frac{1}{\varepsilon}$ of them, again giving an exceptional set of measure $\varepsilon$.

Thus $f:[0,1]^{2} \rightarrow \mathbb{R}$ is continuous, smooth away from the lines $x=j \varepsilon, x=$ $j \varepsilon-\varepsilon^{2}$, satisfies $\partial^{2} f / \partial x \partial y \geq 1$ off these lines and $f(x, y)$ is within $2 \varepsilon$ of $\mathbb{Z}$ except on a set of measure $2 \varepsilon$. Moreover, for each fixed $y, x \mapsto \partial f(x, y) / \partial y$ is continuous where $x=j \varepsilon, x=j \varepsilon-\varepsilon^{2}$. Thus $\partial^{2} f / \partial x \partial y \geq 1$ in the sense of distributions and consequently convolving $f$ with a nonnegative approximate identity on a very small scale (one can quantify this by using Lemma 4.4 to keep track of the widths of the many horizontal exceptional strips occurring) yields the desired $u \in \mathcal{U}_{1,1}$.

Proof of Theorem 4.2. Take $u=u_{\varepsilon}$ as in Lemma 4.3. Then, for $(100 \varepsilon)^{-1}=\lambda \epsilon$ $2 \pi \mathbb{N}$,

$$
\operatorname{Re} I(\lambda)=\int_{F_{\varepsilon}} \cos [\lambda u(x, y)] d x d y+\int_{[0,1]^{2} \backslash F_{\varepsilon}} \cos [\lambda u(x, y)] d x d y .
$$

Now on $F_{\varepsilon}^{C}, \operatorname{dist}(\lambda u(x, y), 2 \pi \mathbb{Z})<2 \varepsilon \lambda=1 / 50$. Hence on $[0,1]^{2} \backslash F_{\varepsilon}, \cos [\lambda u(x, y)] \geq$ $1-\cos (1 / 50)$, and so

$$
\int_{[0,1]^{2} \backslash F_{\varepsilon}} \cos [\lambda u(x, y)] d x d y \geq[1-\cos (1 / 50)][1-10 \varepsilon] .
$$

On the other hand,

$$
\left|\int_{F_{\varepsilon}} \cos [\lambda u(x, y)] d x d y\right| \leq\left|F_{\varepsilon}\right| \leq 10 \varepsilon .
$$

Thus, for $\varepsilon<1 / 100,|I(\lambda)| \geq C_{0}$ where $C_{0}$ is an absolute constant.

We now consider the case where $j$ and $k$ are greater than or equal to one, and at least one of them is at least two. We first establish some lower bounds for $\left\|T_{\lambda}\right\|$.

Lemma 4.5. For $u(x, y)=-(x-y)^{d}(d \geq 2),\left\|T_{\lambda}\right\|_{p \mapsto q} \geq C|\lambda|^{-1 / d}$ for all $p, q$.

Proof. Setting $f \equiv 1$, we see that the size of $T_{\lambda} f(x)$ is essentially $|\lambda|^{-1 / d}$ (either by the one-dimensional van der Corput lemma or by a simple change of variables).

It is known that at least when $d$ is even and $u(x, y)=(x-y)^{d}$, the sharp estimate $\left\|T_{\lambda} f\right\|_{d} \leq C|\lambda|^{-1 / d}\|f\|_{d}$ holds ([JS], [Sj]). This is in spite of the fact that when $d>2, g \mapsto \int_{0}^{1} g\left(x_{1}-t, x_{2}-t^{d}\right) d t$ does not map $L^{d}\left(\mathbb{R}^{2}\right)$ to $L_{1 / d}^{d}\left(\mathbb{R}^{2}\right)[\mathrm{C} 1]$. Note that there is a contrast in $L^{p}$ behaviour between $T_{\lambda}$ and $S_{\alpha}$ for $u(x, y)=(x-y)^{d}$; $S_{\alpha}$ is bounded on all $L^{p}$ with constant $O\left(\alpha^{1 / d}\right)$ while $T_{\lambda}$ is bounded for $p \in\left[d^{\prime}, d\right]$ with constant $O\left(|\lambda|^{-1 / d}\right)$. This is due to the fact that for $T_{\lambda}$ we need to use a result about $\mathcal{U}_{j, k}$ with $j+k=d$ and $1 \leq j, k \leq d-1$, while for $S_{\alpha}$ we can take $0 \leq j, k \leq d$.

Lemma 4.6. Let $u(x, y)=x^{j} y^{k}$ with $j, k \geq 1$ and either $j$ or $k \geq 2$. Then $\left\|T_{\lambda}\right\|_{p \mapsto q} \geq C|\lambda|^{-\left\{\frac{1}{j q} \wedge \frac{1}{k p^{\prime}}\right\}}$. 
Proof. Again set $f \equiv 1$. Then $T_{\lambda} f(x)=\int_{0}^{1} e^{i \lambda x^{j} y^{k}} d y$, which has size

$$
\begin{cases}1, & 0<x<c|\lambda|^{-\frac{1}{j}} \\ \left(|\lambda| x^{j}\right)^{-1}, & c|\lambda|^{-\frac{1}{j}}<x<1\end{cases}
$$

by simple calculations. The $L^{q}$ norm of this function has magnitude $|\lambda|^{-1 / j q}$. By symmetry we also obtain a lower bound of magnitude $|\lambda|^{-1 / k p^{\prime}}$.

Thus we obtain an analogue of Corollary 3.2:

Proposition 4.7. $\sup _{u \in \mathcal{U}_{j, k}}\left\|T_{\lambda}\right\| \geq C \lambda^{-\left\{\frac{1}{j+k} \wedge \frac{1}{j q} \wedge \frac{1}{k p^{\prime}}\right\}}$.

The upper bounds for $\left\|T_{\lambda}\right\|$ that we can obtain are as follows.

Theorem 4.8. Let $k \geq 2$. Then for every $u \in \tilde{\mathcal{U}}_{1, k}$, for every $\lambda \in \mathbb{R}$,

$$
\left\|T_{\lambda}\right\|_{L^{2} \rightarrow L^{2}} \leq C|\lambda|^{-1 / 2 k} \text {. }
$$

Proof. We calculate

$$
\begin{aligned}
\left\|T_{\lambda}^{*} f\right\|_{2}^{2} & =\int\left|\int e^{i \lambda u(x, y)} f(x) d x\right|^{2} d y \\
& =\iiint e^{i \lambda\left[u\left(x_{1}, y\right)-u\left(x_{2}, y\right)\right]} d y f\left(x_{1}\right) \overline{f\left(x_{2}\right)} d x_{2} d x_{1} \\
& =\iint\left\{\int e^{i \lambda\left[u\left(x_{1}, y\right)-u\left(x_{1}+z, y\right)\right]} d y\right\} f\left(x_{1}\right) \overline{f\left(x_{1}+z\right)} d z d x_{1},
\end{aligned}
$$

making the change of variables $z=x_{2}-x_{1}$ for $x_{2}$ with $x_{1}$ fixed. Call $\psi_{z}\left(x_{1}, y\right)=$ $u\left(x_{1}, y\right)-u\left(x_{1}+z, y\right)$ and call $J_{x_{1}, z}(\lambda)=\int e^{i \lambda \psi_{z}\left(x_{1}, y\right)} d y$. Now

$$
\left|\partial_{y}^{k} \psi_{z}\left(x_{1}, y\right)\right|=\left|\int_{x_{1}+z}^{x_{1}} \partial_{s} \partial_{y}^{k} u(s, y) d s\right| \geq|z|
$$

since $u \in \mathcal{U}_{1, k}$. Since $k \geq 2$ here, the classical van der Corput lemma gives

$$
\left|J_{x_{1}, z}(\lambda)\right| \leq C(|\lambda||z|)^{-1 / k} \text {. }
$$

Thus

$$
\left\|T_{\lambda}^{*} f\right\|_{2}^{2} \leq C|\lambda|^{-1 / k} \int|z|^{-1 / k}\left|f\left(x_{1}\right) f\left(x_{1}+z\right)\right| d z d x_{1} \leq C|\lambda|^{-1 / k}\|f\|_{2}^{2},
$$

and so $\left\|T_{\lambda}\right\|_{2-2} \leq C|\lambda|^{-1 / 2 k}$.

For a fixed real-analytic $u$ with critical point at $(0,0)$, satisfying $\partial^{j+k} u / \partial x^{j} \partial y^{k} \neq$ 0 on $Q$, Phong and Stein [PS2] have established the sharp bound $\left\|T_{\lambda}\right\|_{2-2}=$ $O\left(|\lambda|^{-\frac{1}{2(j \vee k)}}\right)$ (among many others). But of course implicit in their result are assumptions on upper bounds of higher derivatives of $u$. On the other hand our result with $j=1$ and $k \geq 2$ is uniform over the class $\mathcal{U}_{1, k}$. When $u \in \mathcal{U}_{j, k}$ with $2 \leq j \leq k$ we are able to make an inductive estimate on $I(\lambda)$ :

Proposition 4.9. For each $j, k$ satisfying $2 \leq j \leq k$, there exists $C<\infty$ such that for every $u \in \tilde{\mathcal{U}}_{j, k}$,

$$
|I(\lambda)| \leq C|\lambda|^{-1 /\left(k \cdot 2^{j}\right)}
$$


As noted above, it follows from this proposition that $\left\|T_{\lambda}\right\|_{2 \mapsto 2} \leq C|\lambda|^{-\alpha}$ for some $\alpha(j, k)>0$.

Proof. We proceed by induction on $j$. The case $j=1$ is given by Theorem 4.8 so we assume that the proposition is true for $2,3, \ldots, j-1$. With $u \in \mathcal{U}_{j, k}$ we begin as in the proof of Theorem 4.8 but with the function $f$ replaced by 1 . Thus

$$
|I(\lambda)|^{2} \leq \int\left|\int e^{i \lambda u(x, y)} d x\right|^{2} d y=\iint J_{x_{1}, z}(\lambda) d z d x_{1}
$$

where $J_{x_{1}, z}(\lambda)=\int e^{i \lambda \psi_{z}\left(x_{1}, y\right)} d y$ and where $\psi_{z}\left(x_{1}, y\right)=u\left(x_{1}, y\right)-u\left(x_{1}+z, y\right)$. We now observe that for $z \leq 0$,

$$
\left(\frac{\partial}{\partial x_{1}}\right)^{j-1}\left(\frac{\partial}{\partial y}\right)^{k} \psi_{z}\left(x_{1}, y\right)=\int_{x_{1}+z}^{x_{1}}\left(\frac{\partial}{\partial s}\right)^{j}\left(\frac{\partial}{\partial y}\right)^{k} u(s, y) d s \geq|z|
$$

since $u \in \mathcal{U}_{j, k}$. There is a similar estimate for $z \geq 0$. Hence

$$
\left|\iint J_{x_{1}, z}(\lambda) d x_{1} d z\right| \leq C|\lambda|^{-1 / 2^{j-1} k}
$$

by the inductive hypothesis (applied on two triangles rather than on $Q$ ). Hence,

$$
|I(\lambda)| \leq C|\lambda|^{-1 /\left(k \cdot 2^{j}\right)},
$$

as desired.

As in subsection 3.1, the estimates on $I(\lambda)$ can be improved if one assumes some additional qualitative condition to be satisfied by $u$. Similar but more complicated results are in $[\mathrm{AKC}]$.

Proposition 4.10. Suppose $j, k \geq 1$, that $u \in \mathcal{U}_{j, k}$ and that in addition, for some constant A,

$$
\text { \# of components of }\left\{y:\left|\frac{\partial^{k} u}{\partial y^{k}}(x, y)\right|<\beta\right\} \leq A
$$

with $A$ independent of $x$ and $\beta$. If $k=1$ we assume also that $\frac{\partial^{2} u}{\partial y^{2}}$ has for each $x$ at most $A$ changes of sign. Then there exists a constant $C_{j, k, A}$ depending only on $j, k$ and $A$ such that for all $|\lambda| \geq 1$,

$$
|I(\lambda)| \leq C_{j, k, A} /|\lambda|^{\frac{1}{j+k}} .
$$

Corollary 4.11. If $u \in \tilde{\mathcal{U}}_{j, k}, j, k \geq 1$ and if for some $N>k, \partial^{N} u / \partial y^{N}$ is singlesigned, or if for some $N>j, \partial^{N} u / \partial x^{N}$ is single-signed, then

$$
|I(\lambda)| \leq C_{N, j, k} /|\lambda|^{\frac{1}{j+k}} .
$$

Proof of Proposition 4.10.

$$
|I(\lambda)| \leq\left|\iint_{\left\{(x, y):\left|\partial_{y}^{k} u\right| \geq \beta\right\}} e^{i \lambda u(x, y)} d y d x\right|+\iint_{\left\{(x, y):\left|\partial_{y}^{k} u\right|<\beta\right\}} d x d y .
$$

For the second term we estimate the inner integral by $C_{j} \beta^{1 / j}$ since $\partial_{x}^{j} \partial_{y}^{k} u \geq 1$. To bound the first, we fix any $x$. Then $\left\{y:\left|\partial^{k} u / \partial y^{k}\right| \geq \beta\right\}$ is by hypothesis a union of at most $A+1$ intervals. The integral of $e^{i \lambda u(x, y)}$ with respect to $y$ over each such 
interval is at most $C_{k}(|\lambda| \beta)^{-1 / k}$ by the classical van der Corput lemma. Thus

$$
|I(\lambda)| \leq C_{j} \beta^{1 / j}+C_{A, k} /(|\lambda| \beta)^{1 / k} .
$$

Optimising in $\beta\left(\beta \approx|\lambda|^{-\frac{j}{j+k}}\right)$ yields the result.

An application of Corollary 4.11 will be given in Section 7 .

The only uniform result (of which the authors are aware) for the operators $T_{\lambda}$ under extra convexity conditions is one of Phong and Stein [PS1]. They show that if $u \in \mathcal{U}_{1,1}$ and satisfies $\partial^{3} u / \partial x \partial y^{2} \geq 0$, then $\left\|T_{\lambda}\right\|_{2-2} \leq C(\log |\lambda|)^{1 / 2} /|\lambda|^{1 / 2}$ with an absolute $C$.

4.2. The higher-dimensional case. We return to the notation of Section 3, in particular of subsection 3.2, and examine $I(\lambda)$ and $T_{\lambda}$ (defined in the obvious way) as an operator from $L^{p}\left(Q^{n^{\prime \prime}}\right)$ to $L^{q}\left(Q^{n^{\prime}}\right)$ where $n^{\prime}+n^{\prime \prime}=n \geq 3$ under the hypothesis $D^{\beta} u \geq 1$ on $Q^{n}$.

There is of course an analogue of Proposition 4.1, with the same proof, which we therefore omit. In analogy with Theorem 4.2, there are no decay estimates for $I(\lambda)$ nor $T_{\lambda}$ if each entry of $\beta$ is 0 or 1 .

Theorem 4.12. For each $n \geq 2$ and each $\beta$ with at least one entry strictly greater than 1 , there exist $\varepsilon=\varepsilon(n, \beta)>0$ and $C=C(\varepsilon, n, \beta)$ such that for every realvalued, integrable function $u$ satisfying $D^{\beta} u \geq 1$ on $Q$, for every $\lambda \in \mathbb{R}, I(\lambda)=$ $\int_{Q} e^{i \lambda u(x)} d x$ satisfies

$$
|I(\lambda)| \leq C|\lambda|^{-\varepsilon} .
$$

Theorem 4.13. For each $n=n^{\prime}+n^{\prime \prime} \geq 2$, each $1 \leq p, q \leq \infty(p \neq 1, q \neq \infty)$ and each $\beta$ with at least one nonzero entry in each of $\left\{1, \ldots, n^{\prime}\right\}$ and $\left\{n^{\prime}+1, \ldots, n\right\}$ and with at least one entry greater than 1 , there exist $\varepsilon=\varepsilon(n, \beta, p, q)>0$ and $C=C(\varepsilon, n, \beta, p, q)$ such that for every real-valued, integrable function u satisfying $D^{\beta} u \geq 1$ on $Q$, for every $\lambda \in \mathbb{R}$, the operator

$$
T_{\lambda} f\left(x^{\prime}\right)=\int_{Q^{n^{\prime \prime}}} e^{i \lambda u\left(x^{\prime}, x^{\prime \prime}\right)} f\left(x^{\prime \prime}\right) d x^{\prime \prime}
$$

satisfies

$$
\left\|T_{\lambda} f\right\|_{L^{q}\left(Q^{n^{\prime}}\right)} \leq C \lambda^{-\varepsilon}\|f\|_{L^{p}\left(Q^{\prime \prime}\right)} .
$$

These two results have already been proved above for $n=2$. Theorem 4.13 follows from Theorem 4.12 by the higher-dimensional analogue of Proposition 4.1.

Proof of Theorem 4.12. The proof is formally the same as that of Proposition 4.9 and is by induction on $n$. The case $n=2$ is contained in Theorem 4.8 and Proposition 4.9 and so we assume the statement of Theorem 4.12 for the case $n-1$. We may assume that an entry of $\beta$ which is at least two occurs among the first $(n-1)$ variables. Let $\beta=\left(\tilde{\beta}, \beta_{n}\right)$. If $\beta_{n}=0$, the statement follows from the inductive hypothesis, by performing the integration in the first $n-1$ variables first. So assume inductively that the statement is true for $\beta_{n} \in\{0,1,2, \ldots, k-1\}$ and that 
$D^{(\tilde{\beta}, k)} u \geq 1$ on $Q$. As in the proof of Proposition 4.9,

$$
\begin{aligned}
|I(\lambda)|^{2} \leq \int_{Q^{n-1}}\left|\int_{Q^{1}} e^{i \lambda u\left(x^{\prime}, y\right)} d y\right|^{2} d x^{\prime} \\
\quad=\iiint e^{i \lambda\left[u\left(x^{\prime}, y\right)-u\left(x^{\prime}, z\right)\right]} d y d z d x^{\prime}=\iiint e^{i \lambda \psi_{s}\left(x^{\prime}, y\right)} d y d x^{\prime} d s
\end{aligned}
$$

where $\psi_{s}\left(x^{\prime}, y\right)=u\left(x^{\prime}, y+s\right)-u\left(x^{\prime}, y\right)=\int_{y}^{y+s} \frac{\partial}{\partial t} u\left(x^{\prime}, t\right) d t$. So

$$
\left|D^{(\tilde{\beta}, k-1)} \psi_{s}\right|=\left|\int_{y}^{y+s} D^{\beta} u\left(x^{\prime}, t\right) d t\right| \geq|s| .
$$

Thus by the inductive hypothesis the inner $d y d x^{\prime}$ integral above is dominated by $(|\lambda||s|)^{-\varepsilon}$, for some $\varepsilon>0$. Hence, by integrating with respect to $s$ we find that $I(\lambda)$ is (uniformly) $O\left(\lambda^{-\varepsilon^{\prime}}\right)$, for some $\varepsilon^{\prime}>0$.

Once again, if we impose some qualitative conditions upon $u$ we can improve the decay estimate for $I(\lambda)$ to the optimal rate; see also [AKC].

Proposition 4.14. Under the hypotheses of Proposition 3.17, there is a constant $C=C\left(\beta, N_{2}, \ldots, N_{n}\right)$ such that

$$
|I(\lambda)| \leq C|\lambda|^{-1 /|\beta|} .
$$

Proof. The proof is the same as that of Proposition 4.10. We write

$$
|I(\lambda)| \leq \int\left|\int_{\left\{x_{n}:\left|\left(\frac{\partial}{\partial x_{n}}\right)^{\beta_{n}} u\right| \geq \gamma\right\}} e^{i \lambda u\left(x^{\prime}, x_{n}\right)} d x_{n}\right| d x^{\prime}+\iint_{\left\{x^{\prime}:\left|\left(\frac{\partial}{\partial x_{n}}\right)^{\beta_{n}} u\right|<\gamma\right\}} d x^{\prime} d x_{n} .
$$

For the second term we use the case $n-1$ of Proposition 3.17 with $\beta^{\prime}$ in place of $\beta$ and $\left(\frac{\partial}{\partial x_{n}}\right)^{\beta_{n}} u$ in place of $u$ to estimate the inner integral by $C \gamma^{1 /\left|\beta^{\prime}\right|}$. For the first we apply the classical van der Corput lemma to estimate the inner integral by $C(|\lambda| \gamma)^{-1 / \beta_{n}}$. Thus $|I(\lambda)| \leq C\left(\gamma^{1 /\left|\beta^{\prime}\right|}+(|\lambda| \gamma)^{-1 / \beta_{n}}\right)$, which, upon taking $\gamma=\lambda^{-\left|\beta^{\prime}\right| /|\beta|}$, yields $|I(\lambda)| \leq C|\lambda|^{-\frac{1}{|\beta|}}$ as desired.

As pointed out in the introduction, most cases of Theorems 3.15 and 3.16 and of Proposition 3.17 are implied by the results of this section, though generally with inferior exponents.

\section{Other SECOND-ORDER DIFFERENTIAL INEQUALITIES}

Let $p(D)$ be a constant-coefficient homogeneous differential operator of degree 2 on $\mathbb{R}^{2}$ with real coefficients. Under what conditions on $p$ is there an implication of the form

$$
p(D) u \geq 1 \text { on } Q \Rightarrow|\{x \in Q:|u(x)| \leq \alpha\}| \leq C \alpha^{\varepsilon}
$$

for some $\varepsilon>0$ and some absolute $C$ ? 
Theorem 5.1. In $\mathbb{R}^{2}, p(D)$ is not elliptic if and only if there exist $\varepsilon, C>0$ such that for every $C^{\infty}$ function $u$ satisfying $p(D) u \geq 1$ on $Q$ and every $\alpha>0$

$$
|\{x \in Q:|u(x)| \leq \alpha\}| \leq C \alpha^{\varepsilon} .
$$

If $p(D)$ is not elliptic, then (5.1) holds for every $\varepsilon<1 / 2$.

Since the measure-theoretic statement involved is invariant under affine changes of variables (except for the domain $Q$, which is immaterial for our purposes) any two $p(D)$ 's related by an invertible linear change of variables will share the same behaviour. Now the symbol of $p(D)$ is merely a quadratic form $p(\xi)$ of two real variables $\xi=\left(\xi_{1}, \xi_{2}\right)$, which can be brought to one of the normal forms $0, \xi_{1}^{2}, \xi_{1} \xi_{2}$ or $\xi_{1}^{2}+\xi_{2}^{2}$ by a linear change of variables. Discarding the trivial case 0 , we see that the cases $\xi_{1}^{2}$ and $\xi_{1} \xi_{2}$ have already been dealt with as $\partial^{2} / \partial x_{1}{ }^{2}$ and $\partial^{2} / \partial x_{1} \partial x_{2}$ respectively, for which there are positive results. Thus Theorem 5.1 follows from the negative result in the special case of the Laplacian $\Delta$ :

Proposition 5.2. For each $0<\delta<1 / 2$, there exists a $u=u_{\delta} \in C^{\infty}(Q)$ such that $\Delta u \equiv 1$ on $Q$ and $|\{x \in Q:|u(x)|<\delta\}| \geq 1-\delta$.

Proof. Fix a compact set $K \subseteq Q^{2}$ with empty interior such that $\mathbb{R}^{2} \backslash K$ is connected and $Q^{2} \backslash K$ has measure $<\delta$ for some pre-assigned $\delta>0$. Let $f(x)=-x_{1}^{2} / 2$. Then by Mergelyan's theorem (see for example [R], p. 423) we may uniformly approximate $f$ on $K$ by a harmonic polynomial $p$, to within $\delta$. Set $u=f-p$. Then $\Delta u \equiv 1$, while on $K,|u(x)|<\delta$. Thus $|\{x \in Q:|u(x)|<\delta\}| \geq 1-\delta$ since $|K| \geq 1-\delta$.

The same considerations establish that the only homogeneous second order constant coefficient differential operators $p(D)$ in $n$ dimensions which possess favourable measure estimates for the sublevel set of $u$ 's with $p(D) \geq 1$ are those which can be written as compositions of two first order directional derivatives. We do not know whether a similar result is true for homogeneous constant coefficient differential operators of arbitrary order. We wonder whether a more general setting for these inequalities might be in terms of compositions of vector fields; such a formulation might be invariant under diffeomorphism, whereas the setup for our results is strongly coordinate-dependent.

\section{Connections With combinatorial problems}

The questions addressed in this paper - in particular the sublevel set estimates of Section 3 - have connections with certain combinatorial problems arising in extremal graph theory. A continuous analogue of such a problem is as follows:

Problem 6.1. Suppose $E \subseteq Q=[0,1]^{2}$ has Lebesgue measure $|E|>0$. Is it true that for some absolute constant $\varepsilon_{0}$, one can always find four points $A, B, C, D \in E$, such that the quadrilateral figure $A B C D$ is a rectangle with sides parallel to the coordinate axes, whose area is at least $\varepsilon_{0}|E|^{2}$ ?

This problem has little to do with Lebesgue measure, and can be couched in terms of probability measures on $\mathbb{R}$.

Problem 6.2. Let $\mu, \nu$ be probability measures on $\mathbb{R}$. Suppose $E \subseteq \mathbb{R}^{2}$ satisfies $\mu \otimes \nu(E)>0$. Is there an absolute constant $\varepsilon_{0}$ (independent of $E$ ), such that one can always find four points $A, B, C, D \in E$, such that the quadrilateral $A B C D$ is a rectangle $R$ with sides parallel to the coordinate axes, and such that

$$
(\mu \otimes \nu)(R) \geq \varepsilon_{0}(\mu \otimes \nu)(E)^{2} ?
$$


Specialisation of Problem 6.2 to counting measure on $[1, N]$ gives rise to a matrix problem:

Problem 6.3. Does there exist $\varepsilon_{0}>0$ such that for any $M, N$, for any $N \times N$ matrix $A$ at least $M$ of whose entries are equal to $1, A$ has a $2 \times 2$ submatrix all of whose entries are equal to 1 , and whose area is at least $\varepsilon_{0} M^{2} / N^{2}$ ?

Nets Katz has shown that if there is such an $\varepsilon_{0}$, then it cannot exceed $1 / 5$.

If Problem 6.1 has an affirmative answer, then every $u \in \mathcal{U}_{1,1}$ would satisfy $\left|\left\{x \in Q^{2}:|u(x)| \leq \alpha\right\}\right| \leq C \alpha^{1 / 2}$ for some absolute $C$. That is, the logarithmic factor occurring in Corollary 3.5 would be superfluous. Indeed, the proof of Lemma 3.6 shows that if $u \in \mathcal{U}_{1,1}$ and if $E=\{(x, y):|u(x, y)| \leq \alpha\}$, then $\left(x_{i}, y_{i}\right) \in E$ for $i, j \in\{0,1\}$ implies that the area of the rectangle with vertices $\left(x_{i}, y_{j}\right), i, j \in\{0,1\}$, is at most $4 \alpha$. The contrapositive of the (putative) positive solution to Problem 6.1 would then force $E$ to have area at most $C \alpha^{1 / 2}$. On the other hand, by re-working the methods of Section 3, one can show:

Proposition 6.1. There exists an absolute constant $\varepsilon_{0}>0$ with the following property. Let $\mu, \nu$ be probability measures on $\mathbb{R}$. For any set $E \subseteq \mathbb{R}^{2}$ satisfying $\mu \otimes \nu(E)>0$, there exists a rectangle $R \subseteq \mathbb{R}^{2}$ whose vertices all belong to $E$, satisfying $^{6}(\mu \otimes \nu)(R) \geq \varepsilon_{0}(\mu \otimes \nu)(E)^{2} / \log ^{+}[1 /(\mu \otimes \nu)(E)]$.

Here $\log ^{+}(t)=\max (1, \log (t))$.

Proof. We prove the contrapositive statement: if every rectangle $R$ (with sides parallel to the axes) with vertices in $E$ has $(\mu \otimes \nu)(R) \leq \alpha$, then $(\mu \otimes \nu)(E) \leq$ $C \alpha^{1 / 2} \log (1 / \alpha)^{1 / 2}$. Indeed, as in the proof of Theorem 3.4,

$$
\begin{aligned}
(\mu \otimes \nu)(E)^{2} & \leq \iint \mu\left(E\left(y_{1}\right) \cap E\left(y_{2}\right)\right) d \nu\left(y_{1}\right) d \nu\left(y_{2}\right) \\
& \leq 2 \iint_{y_{2} \geq y_{1}}\left[\frac{\alpha}{\nu\left(\left(y_{1}, y_{2}\right]\right)} \wedge 1\right] d \nu\left(y_{1}\right) d \nu\left(y_{2}\right) \\
& \leq C \alpha \log (1 / \alpha) .
\end{aligned}
$$

There are also combinatorial problems featuring more general polygons than rectangles. These arise typically as follows. (Incidentally, what follows gives an alternative proof of Lemma 3.6; Lemma 3.8 can likewise be obtained from the same idea.)

Lemma 6.2. Suppose $\partial^{2} u / \partial x \partial y \geq 1$ on $Q$. Suppose that $\Gamma \subseteq Q$ is a polygonal Jordan curve, all of whose edges are either horizontal or vertical. Assume that each corner of $\Gamma$ belongs to $E=\{(x, y):|u(x, y)| \leq \alpha\}$. Let $R$ be the region enclosed by Г. Then

$$
\operatorname{Area}(R) \leq \#(\text { corners of } \Gamma) \cdot \alpha .
$$

By a corner of $\Gamma$ we mean of course a point where a horizontal segment and a vertical segment of $\Gamma$ meet.

\footnotetext{
${ }^{6}$ If $R$ is a rectangle with vertices $\left(x_{i}, y_{j}\right), i, j \in\{0,1\}$, then $\mu \otimes \nu(R)$ is defined as $\mu\left(\left(x_{0}, x_{1}\right]\right) \nu\left(\left(y_{0}, y_{1}\right]\right)$.
} 
Proof. By Stokes' theorem,

$$
\int_{R} u_{x y} d x d y=\frac{1}{2} \int_{\Gamma}\left(u_{x} d x-u_{y} d y\right)= \pm\left[u\left(v_{1}\right)-u\left(v_{2}\right)+u\left(v_{3}\right) \ldots\right]
$$

where $v_{1}, v_{2}, \ldots$ are the corners of $\Gamma$ in order.

Thus an affirmative answer to the following question would suffice to remove the logarithmic term from Corollary 3.5.

Problem 6.4. Suppose $E \subseteq Q^{2}$ has Lebesgue measure $|E|>0$. Is it true that for some absolute constant $\varepsilon_{0}$ one may find $k \in\{4,6,8, \ldots\}$ and points $V_{1}, V_{2}, \ldots, V_{k}$ in $E$ such that the figure $V_{1} V_{2} \ldots V_{k}=R$ is a closed Jordan curve with all edges either horizontal or vertical, and is such that

$$
\operatorname{area}(R) \geq \varepsilon_{0} k|E|^{2} ?
$$

Nets Katz $[\mathrm{K}]$ has solved a modified version of Problem 6.4. He produces a closed curve with sides parallel to the coordinate axes, having either four or six corners and satisfying the desired area estimate; however, in the case of six corners, the curve may have self-crossings (which are not counted among its corners).

A hierarchy of similar combinatorial problems arises in connection with the sublevel set problem for $\mathcal{U}_{j, k}$ in $\mathbb{R}^{2}$ and for $\mathcal{U}_{\beta}$ in $\mathbb{R}^{n}, n \geq 3$. Thus, for example, the sublevel set problem for $\mathcal{U}_{(1,1, \ldots, 1)}$ in $\mathbb{R}^{n}$ - for which Theorem 3.15 gives an estimate of $O\left(\varepsilon^{\frac{1}{2^{n-1}}}(\log 1 / \varepsilon)^{\gamma_{n}}\right)$ for some $\gamma_{n}>0$ - leads to the variant of Problem 6.1 in $Q^{n}$ where quadrilateral figures in $Q^{2}$ are replaced by rectangles in $Q^{n}$ with sides parallel to the axes. Keleti $[\mathrm{Ke}]$ has observed that one can find such a rectangle with volume $\geq \varepsilon_{0}|E|^{2^{n-1}} \cdot\left(\log ^{+} 1 /|E|\right)^{-\gamma_{n}}$, the same bound which is implied by our analysis in Theorem 3.15. He has also observed that, if a conjecture of Erdös $[E]$ is true, then modulo the logarithmic factors this combinatorial result would be sharp.

\section{Polynomials of Bounded degree}

In this section we study the special case where $u$ is a polynomial whose degree does not exceed some pre-assigned, but arbitrary, quantity. Here supplementary convexity hypotheses of the type imposed in Proposition 3.17 are automatically satisfied. Consequently we obtain:

Theorem 7.1. For each $d, n$ there exists a finite constant $C$ such that for any multi-index $\beta$ and any polynomial $u: \mathbb{R}^{n} \rightarrow \mathbb{R}$ of degree $\leq d$ satisfying $D^{\beta} u(x) \geq 1$ for every $x \in Q^{n}$,

$$
\left|\left\{x \in Q^{n}:|u(x)| \leq \alpha\right\}\right| \leq C \alpha^{1 /|\beta|} .
$$

The weaker conclusion with exponent $1 / d$ instead of $1 /|\beta|$ may easily be proved by a slicing argument, as for instance in the proof of Lemma 3.4 of [C2]. This theorem can be used to sharpen some results of Ricci and Stein [RS].

As a direct consequence of Proposition 4.14, we have a van der Corput lemma for polynomials in higher dimensions, which can also be obtained by the methods of $[\mathrm{AKC}]$. 
Theorem 7.2. For each $d, n$ there exists $C$ such that for any polynomial $u: Q^{n} \rightarrow$ $\mathbb{R}$ of degree $\leq d$ which for some multi-index $\beta$ satisfies $D^{\beta} u(x) \geq 1$ for every $x \in Q^{n}$,

$$
\left|\int_{Q^{n}} e^{i \lambda u(x)} d x\right| \leq C|\lambda|^{-1 /|\beta|}
$$

Corollary 7.3. Let $p(t)=\sum_{|\alpha| \leq d} c_{\alpha} t^{\alpha}, c_{\alpha} \in \mathbb{R}$. Then

$$
\left|\int_{Q^{n}} e^{i p(t)} d t\right| \leq C_{d, n}\left(\sum_{0<|\alpha| \leq d}\left|c_{\alpha}\right|\right)^{-1 / d}
$$

Moreover $C_{d, 1} \leq C d$ for an absolute constant $C$.

Proof. Without loss of generality $p(0)=0$. Let $\mathcal{P}_{d}$ denote the vector space of all polynomials $p(t)=\sum_{0<|\alpha| \leq d} c_{\alpha} t^{\alpha}$, equipped with the norm $\|p\|=\sum_{0<|\alpha| \leq d}\left|c_{\alpha}\right|$. As in [CRW], consider the functional

$$
\theta(p)=\max _{0<|\alpha| \leq d} \inf _{t \in Q^{n}}\left|D^{\alpha} p(t)\right| .
$$

This is a continuous function on $\mathcal{P}_{d}$, which is homogeneous of degree 1 . Moreover $\theta(p) \neq 0$ for $0 \neq p \in \mathcal{P}_{d}$; vanishing would force the coefficients of $p$, starting with the highest order ones, to all be zero. Thus, for some constant depending only on $n$ and $d, \theta(p) \geq C_{d, n}^{\prime}\|p\|$ for all $p \in \mathcal{P}_{d}$. Theorem 7.2 now yields the desired conclusion.

This could alternatively be derived directly from the one-dimensional case, using the functional

$$
\theta(p)=\sup _{\mu, \nu} \inf _{t \in Q^{n}}\left|(\nu \cdot \nabla)^{\mu} p(t)\right|,
$$

where $\mu \in\{1, \ldots, d\}$ and $\nu$ represents an arbitrary unit vector in $\mathbb{R}^{n}$. The details are left to the reader.

To obtain the linear bound for $C_{d, 1}$ we need the following variant of the argument, which together with a simple rescaling allows us to apply Proposition 2.2 to complete the proof.

Lemma 7.4. Let $p(t)=\sum_{j=1}^{d} c_{j} t^{j}$. Suppose $\sum_{j=1}^{d}\left|c_{j}\right| \geq$ e. Then, for some $j,\left|p^{(j)}(t)\right| \geq$ $\frac{1}{2}$ on $I=\left[0, \log _{e} \frac{3}{2}\right]$.

Proof. Consider the statement, for $j=-1,0,1,2, \ldots, d-2$, that

$$
\max \left\{d !\left|c_{d}\right|, \ldots,(d-j) !\left|c_{d-j}\right|\right\} \leq 1 \text { and }\left|c_{d-j-1}\right| \geq \frac{1}{(d-j-1) !} .
$$

(When $j=-1$ the statement is simply $\left|c_{d}\right| \geq \frac{1}{d !}$.) If for some $j$ this is true, we are done because then

$$
p^{(d-j-1)}(t)=(d-j-1) ! c_{d-j-1}+\frac{(d-j) !}{1 !} c_{d-j} t+\cdots+\frac{d !}{(j+1) !} c_{d} t^{j+1}
$$

satisfies

$$
\left|p^{(d-j-1)}(t)\right| \geq 1-\left\{t+\frac{t^{2}}{2 !}+\cdots+\frac{t^{j+1}}{(j+1) !}\right\} \geq 1-\left(e^{t}-1\right) \geq \frac{1}{2}
$$


if $e^{t} \leq 3 / 2$, that is, if $t \in I$. If the statement fails for all $j$, we have $\left|c_{d}\right| \leq$ $\frac{1}{d !},\left|c_{d-1}\right| \leq \frac{1}{(d-1) !}, \ldots,\left|c_{2}\right| \leq \frac{1}{2 !}$ and $\left|c_{1}\right| \leq \frac{1}{1 !}$, which contradicts $\sum_{j=1}^{d}\left|c_{j}\right| \geq e$.

Consider the operators $T_{\lambda} f(x)=\int_{Q^{n^{\prime \prime}}} e^{i \lambda q(x, y)} f(y) d y$ where $n=n^{\prime}+n^{\prime \prime}$ and $(x, y) \in Q^{n^{\prime}} \times Q^{n^{\prime \prime}}$.

Corollary 7.5. For each $d, n$ and each pair of exponents $1 \leq p, q \leq \infty$ with $p \neq 1$ and $q \neq \infty$, there exist $C<\infty$ and $\delta>0$ such that for any polynomial $q(x, y)=$ $\sum_{\alpha} c_{\alpha} x^{\alpha^{\prime}} y^{\alpha^{\prime \prime}}$ of degree $\leq d$

$$
\left\|T_{\lambda}\right\|_{L^{p} L^{q}} \leq C \lambda^{-\delta}\left(\sum_{\alpha^{\prime} \neq 0}\left|c_{\alpha}\right|\right)^{-\delta} .
$$

Proof. By interpolation it suffices to prove this for $p=q=2$. It is no loss of generality to assume that $\sum_{\alpha^{\prime} \neq 0 \text { and } \alpha^{\prime \prime} \neq 0}\left|c_{\alpha}\right|=1$. Fix $\beta$ with $\beta^{\prime}, \beta^{\prime \prime} \neq 0$ such that $\left|c_{\beta}\right| \sim 1$. One has

$$
\left\|T_{\lambda} f\right\|_{2}^{2}=\iint_{Q^{n^{\prime \prime}} \times Q^{n^{\prime \prime}}} K(y, z) f(y) \bar{f}(z) d y d z
$$

where

$$
K(y, z)=\int_{Q^{n^{\prime}}} e^{i \lambda[q(x, y)-q(x, z)]} d x
$$

By Corollary 7.3,

$$
|K(y, z)| \leq C \min \left(1, \lambda^{-1 / d}|\rho(y, z)|^{-1 / d}\right)
$$

where $\rho(y, z)=\left.\partial_{x}^{\beta^{\prime}}[q(x, y)-q(x, z)]\right|_{x=0}$. Therefore it suffices to show that for some $C<\infty$ and $\delta>0$ depending only on $n, d$,

$$
\sup _{y} \int_{Q^{n^{\prime \prime}}}|\rho(y, z)|^{-\delta} d z \leq C
$$

As a function of $z, \rho$ is a polynomial of degree $\leq d$ for each $y$, and $\left|\partial_{z}^{\beta^{\prime \prime}} \rho(y, 0)\right| \sim 1$. Therefore (7.1) follows from the sublevel set version of Corollary 7.3, which is a lemma established in [RS], and which could alternatively be deduced from various results in this paper.

\section{Applichtions}

Results in the previous section can be used to simplify the proof of a theorem of Ricci and Stein [RS] and to improve their estimate in the one-dimensional case. Let $T$ be a bounded Calderón-Zygmund operator, associated in the usual sense to a kernel $K(x, y)$, and let $q$ be a real-valued polynomial. Then there is an operator $\tilde{T}_{q}$ associated to the kernel $e^{i q(x, y)} K(x, y)$, defined for test functions $f$ by

$$
\tilde{T}_{q} f(x)=e^{i q(x, x)} T f(x)+\int\left(e^{i q(x, y)}-e^{i q(x, x)}\right) K(x, y) f(y) d y
$$


Theorem 8.1. Let $T$ be a Calderón-Zygmund operator that is bounded on $L^{2}\left(\mathbb{R}^{n}\right)$. Then for each degree $d \geq 1$ and each $p \in(1, \infty)$ there exists $C=C(n, d, p, T)<\infty$ such that for every real-valued polynomial $q$ of degree at most $d$,

$$
\left\|\tilde{T}_{q}\right\|_{L^{p} L^{p}} \leq C .
$$

Moreover when $n=1$ we may take $C \leq C^{\prime} \cdot d$ where $C^{\prime}$ is independent of the degree $d$.

The original proof of [RS] relied on an induction on the degree of $q$; with Corollary 7.5 in hand this induction can be eliminated. Otherwise the proof is essentially the same. The details are left to the interested reader.

Remark. It would be interesting to determine the true behaviour of $C_{1, d, p}$ as a function of $d$. Certain examples suggest that $C_{1, d, p} \geq c_{p} \log (d+2)$, but we have not verified this in detail.

Our next application contains all but the nondegenerate case of Pan's generalisation $[\mathrm{P}]$ of Theorem 8.1, and also applies in certain 'flat' cases where Pan's hypothesis is not valid.

Theorem 8.2. Let $V$ be a small neighbourhood of $(0,0) \in \mathbb{R}^{n} \times \mathbb{R}^{n}$ and let $u: V \rightarrow$ $\mathbb{R}$ be smooth. Let $\beta=\left(\beta_{1}, \beta_{2}\right) \in \mathbb{N}^{n} \times \mathbb{N}^{n}$ Suppose that both $\beta_{1}, \beta_{2}$ are nonzero and that at least one coordinate of $\beta_{1}$ or $\beta_{2}$ is strictly greater than one. Suppose there exists a constant $A$ so that for all $s \in(0,1)$

$$
\max _{\gamma:|\gamma|=|\beta|} \sup _{\substack{|x-y| \leq s \\(x, y) \in V}}\left|D^{\gamma} u(x, y)\right| \leq A \inf _{\substack{|x-y| \geq s \\(x, y) \in V}}\left|D^{\beta} u(x, y)\right| .
$$

Let $K$ be the kernel of an $L^{2}$-bounded Calderón-Zygmund operator on $\mathbb{R}^{n}$. Then for any $\phi \in C_{0}^{\infty}(V)$, the operators $\tilde{T}_{\lambda u}$ are bounded on $L^{p}(\mathbb{R})$ for $1<p<\infty$, uniformly in $\lambda \in \mathbb{R}$.

Proof. For simplicity of notation we shall assume that $K$ is a convolution kernel and is homogeneous. Without loss of generality $u(x, x) \equiv 0$. Fix an $\alpha<1$ to be determined later, and consider the operators $T_{\lambda}^{0}$ and $T_{\lambda}^{j}, j \geq 1$, obtained by truncating smoothly the kernels of $T_{\lambda}$ at $|x-y| \leq \alpha$ and at $|x-y| \sim 2^{j} \alpha$ respectively.

Now $T_{\lambda}^{j}$ has the same operator norms as the operator with kernel

$$
e^{i \lambda u\left(2^{j} \alpha x, 2^{j} \alpha y\right)} K(x, y) \chi_{|x-y| \sim 1} \phi\left(2^{j} \alpha x, 2^{j} \alpha y\right),
$$

for which it suffices to estimate the operator norms corresponding to the kernels

$$
e^{i \lambda u\left(2^{j} \alpha[x+\ell], 2^{j} \alpha[y+\ell]\right)} K(x, y) \chi_{|x-y| \sim 1} \chi_{|y| \leq 1} \phi\left(2^{j} \alpha[x+\ell], 2^{j} \alpha[y+\ell]\right)
$$

uniformly in $\ell \in \mathbb{Z}^{n}$.

When $|x-y| \sim 1$, we have

$$
\begin{aligned}
\left|D^{\beta}\left\{\lambda u\left(2^{j} \alpha[x+\ell], 2^{j} \alpha[y+\ell]\right)\right\}\right| & =|\lambda|\left(2^{j} \alpha\right)^{|\beta|}\left|D^{\beta} u\left(2^{j} \alpha[x+\ell], 2^{j} \alpha[y+\ell]\right)\right| \\
& \geq|\lambda|\left(2^{j} \alpha\right)^{|\beta|} \inf _{|x-y| \geq \alpha}\left|D^{\beta} u\right| .
\end{aligned}
$$

Hence by Theorem 4.13

$$
\left\|\sum_{j \geq 1} T_{\lambda}^{j}\right\|_{p-p} \leq C\left\{|\lambda| \alpha^{|\beta|} \inf _{|x-y| \geq \alpha}\left|D^{\beta} u\right|\right\}^{-\varepsilon}
$$

for some $\varepsilon>0$. 
For the local part, $T_{\lambda}^{0}$, we follow Pan's approach in $[\mathrm{P}]$. It suffices to show that the operators $T_{\lambda}^{0 \ell}, \ell \in \mathbb{Z}^{n}$, with kernels

$$
e^{i \lambda u(x, y)} K(x, y) \phi(x, y) \chi|x-y| \leq \alpha \chi|x-\alpha \ell| \leq \alpha
$$

have uniform bounds in $\ell$. For each such $\ell$ we first expand $u(x, y)$ in a Taylor series in $y$ about $x$ (with $|x-y| \leq \alpha$ ) to obtain, for any $M \geq 2$,

$$
u(x, y)=\sum_{1 \leq|\gamma|<M} \frac{(y-x)^{\gamma}}{\gamma !} D_{y}^{\gamma} u(x, x)+O\left(|x-y|^{M} \sup _{|\gamma|=M|\zeta-\eta| \leq \alpha} \sup _{\mid y}\left|D_{y}^{\gamma} u(\zeta, \eta)\right|\right)
$$

and then expand each $D_{y}^{\gamma} u(x, x)$ about $x=\alpha \ell$ with $|x-\alpha \ell| \leq \alpha$ to obtain, for $N_{\gamma}:=M-|\gamma|$

$$
\begin{aligned}
D_{y}^{\gamma} u(x, x)= & \sum_{|\sigma|<N_{\gamma}} \frac{(x-\alpha \ell)^{\sigma}}{\sigma !} \mathcal{D}^{(\sigma, \gamma)} u(\alpha \ell, \alpha \ell) \\
& +O\left(|x-\alpha \ell|^{N_{\gamma}} \sup _{|\tau|=N_{\gamma}|\theta-\alpha \ell| \leq \alpha} \sup _{\left.\left|\mathcal{D}^{(\tau, \gamma)} u(\theta, \theta)\right|\right)}\right.
\end{aligned}
$$

where $\mathcal{D}^{(\sigma, \gamma)}$ is some constant coefficient differential operator of order $|\sigma|+|\gamma|$. Combining these equations, for each $\ell \in \mathbb{Z}^{n}, M$ and $\left\{N_{\gamma}:|\gamma|<M\right\}$, we find that $u(x, y)$ equals a polynomial $q(x, y)$, depending on $\ell, M$ but having uniformly bounded degree, plus

$$
O\left(|x-y|^{M}+\sum_{1 \leq|\gamma|<M}|x-y|^{|\gamma|}|x-\alpha \ell|^{N_{\gamma}}\right) \sup _{|\gamma|=M|x-y| \leq \alpha} \sup _{\left|{ }^{\prime}\right| \leq \alpha}\left|D^{\gamma} u(x, y)\right| .
$$

Now the operators $S_{\lambda}^{\ell}$ with kernels

$$
e^{i \lambda q(x, y)} K(x, y) \phi(x, y) \chi_{|x-y| \leq \alpha} \chi_{|x-\alpha \ell| \leq \alpha}
$$

do have uniform $L^{p}$ bounds in $\lambda, \ell, \alpha$ (and $M$ ) by Theorem 8.1. So it suffices to control $T_{\lambda}^{0, \ell}-S_{\lambda}^{\ell}$, whose kernel we can dominate by

$$
\begin{aligned}
|\lambda|\left[|x-y|^{M-n}\right. & \left.+\sum_{1 \leq \gamma<M}|x-y|^{|\gamma|-n}|x-\alpha \ell|^{N_{\gamma}}\right] \chi_{|x-y| \leq \alpha} \chi_{|x-\alpha \ell| \leq \alpha} \\
& \cdot \sup _{|\gamma|=M} \sup _{|x-y| \leq \alpha}\left|D^{\gamma} u(x, y)\right| .
\end{aligned}
$$

Hence $T_{\lambda}^{0, \ell}-S_{\lambda}^{\ell}$ is $L^{p}$ bounded, $1 \leq p \leq \infty$, with a constant dominated by

$$
|\lambda| \alpha^{M} \sup _{|\gamma|=M} \sup _{|x-y| \leq \alpha}\left|D^{\gamma} u(x, y)\right| .
$$

Combining all the estimates gives

$$
\left\|T_{\lambda}\right\|_{p-p} \leq C+\frac{C}{\left\{|\lambda| \alpha^{|\beta|} \inf _{|x-y| \geq \alpha}\left|D^{\beta} u\right|\right\}^{\varepsilon}}+C \lambda \alpha^{M} \sup _{|\gamma|=M|x-y| \leq \alpha} \sup _{\left|x^{\gamma} u\right|} \mid D^{\gamma}
$$

for $1<p<\infty$. Choosing $M=|\beta|$, applying the hypothesis (8.1) and then choosing $\alpha$ so that $|\lambda| \alpha^{|\beta|} \inf _{|x-y| \geq \alpha}\left|D^{\beta} u\right| \approx 1$ concludes the proof.

Note that if $\beta$ is as in the hypothesis of the theorem, and if $u$ is smooth and $D^{\beta} u(0,0) \neq 0$, then hypothesis (8.1) is trivially satisfied if $V$ is taken to be sufficiently small. This situation corresponds to Pan's case $[\mathrm{P}]$. However hypothesis (8.1) may also be satisfied even when $D^{\beta} u(0,0)=0$ for all $\beta$. For example if $n=1$ and $u(x, y)=\gamma(x-y)$, then (8.1) is satisfied if $\gamma^{(j)}$ is increasing for some $j \geq 2$. 
When $n=1$, examples such as $u(x, y)=e^{-\psi(x, y)(x-y)^{-2}}$ (where $\psi \in C^{\infty}$ and $\psi(x, x)>0)$ also satisfy condition (8.1).

One can also obtain results for (certain semi-translation invariant) singular Radon transforms associated to families of hypersurfaces by a direct application of Plancherel's theorem. We merely state the result corresponding to Theorem 8.2:

Theorem 8.3. Under the same hypotheses as Theorem 8.2, for any CalderónZygmund convolution kernel $K$ on $\mathbb{R}^{n}$, the operator $S$ given by

$$
S g\left(x, x_{n+1}\right)=\int_{\mathbb{R}^{n}} g\left(x-y, x_{n+1}-u(x, x-y)\right) \phi(x, x-y) K(y) d y
$$

is bounded on $L^{2}\left(\mathbb{R}^{n+1}\right)$.

Similarly, all the $L^{2}-L^{2}$ estimates for $T_{\lambda}$ in Section 4 give corresponding uniform $L^{2}$ smoothing estimates, with respect to the last coordinate, for certain semi-translation invariant Radon transforms; these may then by interpolated to obtain $L^{p}$ smoothing. Denote by $\mathcal{L}_{\varepsilon}^{p}\left(\mathbb{R}^{n+1}\right)$ the space of functions $h$ such that $\left[\left(1+\left|\xi_{n+1}\right|^{2}\right)^{\frac{\varepsilon}{2}} \hat{h}\right]^{\vee}$ belongs to $L^{p}\left(\mathbb{R}^{n+1}\right)$. Then corresponding to Theorem 4.13 and Proposition 4.9, we have:

Theorem 8.4. Let $n \geq 1$, and let $\beta=\left(\beta_{1}, \beta_{2}\right) \in \mathbb{N}^{n} \times \mathbb{N}^{n}$ with both $\beta_{1}, \beta_{2}$ nonzero and at least one coordinate of one $\beta_{j}$ strictly greater than one. Suppose $D^{\beta} u \geq 1$ on $Q^{2 n}$. Define, for $\phi \in C_{c}^{\infty}\left(Q^{2 n}\right)$

$$
S g\left(x, x_{n+1}\right)=\int_{\mathbb{R}^{n}} g\left(x-y, x_{n+1}-u(x, x-y)\right) \phi(x, y) d y .
$$

Then, for each $p \in(1, \infty)$ there exists an $\varepsilon=\varepsilon(n, \beta, p)>0$ and $a C=C(\varepsilon, n, \beta, p, \phi)$ such that

$$
\|S g\|_{\mathcal{L}_{\varepsilon}^{p}\left(\mathbb{R}^{n+1}\right)} \leq C\|g\|_{L^{p}\left(\mathbb{R}^{n+1}\right)} .
$$

Moreover when $n=2, p=2$ and $\beta=(1, k)$ with $k \geq 2$, we may take $\varepsilon=1 / 2 k$.

Remark. The original motivation for the study of the problems described above aside from their intrinsic interest - arose in the study of singular integrals along flat curves, undertaken by the first and last authors in collaboration with Stephen Wainger. To oversimplify matters a bit, one sought $L^{2}$ operator smoothing estimates for certain operators $T_{j}$ associated to appropriately normalised pieces of the curve in question; the method to be applied dictated the need for uniformity in the estimates over $j$. It involved writing out the kernels $K_{j}$ of the $T_{j}$ explicitly and looking for $L^{1}$ smoothness of these $K_{j}$. To a first approximation, the expressions to be controlled then took the form $\int_{Q^{3}} u_{j}(x)^{-1} d x$, where the only useful information available about $\left\{u_{j}\right\}$ was that $\partial^{3} u_{j} / \partial x^{2} \partial y \geq 1$ uniformly in $j$. Thus the problem addressed by Theorem 1.3 arose. This motivating problem was eventually circumvented by other methods - see [CWW].

In the case $n=1$, results more general than Theorem 8.3 in the flat setting had been given by [Se1], and further results have been obtained in [CP]. It would be interesting to apply the results of this paper to operators further from the Euclideantranslation-invariant case (e.g. to operators associated to flat vector fields [CSWW] or the Heisenberg group [CWW]), in the spirit of their original motivation. 


\section{A REMARK ON MULTI-SUBLEVEL SETS}

Here we discuss the sublevel set problem for a function $\phi$ in the intermediate case where $\partial^{\alpha} \phi$ is assumed to be $C^{\infty}$, but $\phi$ itself is merely integrable. Whereas it is well-known that estimates for oscillatory integrals of the first kind, $\int \exp (i \lambda \psi)$, lead to estimates for sublevel sets, we will exploit instead estimates for oscillatory integral operators, $\int \exp (i \lambda \psi(x, y)) F(x) G(y) d x d y$.

The following remarks originated in a conversation between the authors and T. Tao.

A collection $\left\{r_{j}\right\}$ of numbers is said to be $\delta$-separated if $\left|r_{j}-r_{k}\right| \geq \delta$ for all $j \neq k$.

Proposition 9.1. Suppose that $\phi:[0,1]^{2} \mapsto \mathbb{R}$ is integrable, and that $\partial^{2} \phi / \partial x \partial y$ is $C^{\infty}$ and nowhere vanishing. Then there exists $C<\infty$ such that for any $\delta>0$, any $1 \leq N<\infty$, and any collection $\left\{r_{1}, \ldots, r_{N}\right\}$ of $\delta$-separated numbers,

$$
\left|\bigcup_{j=1}^{N}\left\{(x, y):\left|\phi(x, y)-r_{j}\right|<\delta\right\}\right| \leq C N^{1 / 2} \sqrt{\log (1+N)} \cdot \delta^{1 / 2}
$$

In particular, taking $N=1$, we see that the factor $\sqrt{\log \left(\delta^{-1}\right)}$ in Corollary 3.5 can be dispensed with here. The example $\phi(x, y)=(x-y)^{2}$ demonstrates that this dependence on $\delta, N$ is optimal, except possibly for the logarithmic factor.

Proof. While $\phi$ itself is not assumed to be smooth, we can decompose $\phi(x, y)=$ $\psi(x, y)+f(x)+g(y)$, where $\partial^{2} \phi / \partial x \partial y \equiv \partial^{2} \psi / \partial x \partial y$, and $\psi \in C^{\infty}$. Fix such a real-valued decomposition.

Then by a theorem of Hörmander $[\mathrm{H}]$,

$$
\left|\int_{[0,1]^{2}} e^{i \lambda \psi(x, y)} F(x) G(y) d x d y\right| \leq C(1+|\lambda|)^{-1 / 2}\|F\|_{L^{2}([0,1])}\|G\|_{L^{2}([0,1])}
$$

for all $F, G \in L^{2}$ and all $\lambda \in \mathbb{R}$. Taking $F=\exp (i \lambda f)$ and $G=\exp (i \lambda g)$ yields

$$
\left|\int_{[0,1]^{2}} e^{i \lambda \phi(x, y)} d x d y\right| \leq C(1+|\lambda|)^{-1 / 2} .
$$

Set $E_{j}=\left\{(x, y):\left|\phi(x, y)-r_{j}\right|<\delta\right\}$. Fix a nonnegative auxiliary function $h \in C_{0}^{\infty}(\mathbb{R})$, such that $h(t) \equiv 1$ for $|t| \leq 1$. If $\left|\phi(x, y)-r_{j}\right|<\delta$ for some $j$, then $h\left(\left[\phi(x, y)-r_{j}\right] / \delta\right)=1$. Thus

$$
\left|\bigcup_{j} E_{j}\right| \leq\left|\int_{[0,1]^{2}} \sum_{j=1}^{N} h\left(\frac{\phi(x, y)-r_{j}}{\delta}\right) d x d y\right| .
$$

Writing $h$ as the inverse Fourier transform of its Fourier transform, and setting $\lambda=\delta^{-1}$,

$$
\left|\bigcup_{j} E_{j}\right| \leq C\left|\int_{-\infty}^{\infty} \hat{h}(\xi) \int_{[0,1]^{2}} \sum_{j=1}^{N} e^{i \lambda\left(\phi(x, y)-r_{j}\right) \xi} d x d y d \xi\right|=C\left|\int_{-\infty}^{\infty} \hat{h}(\xi) v(\xi) u(\xi) d \xi\right|
$$

where $u(\xi)=\sum_{j} e^{-i \lambda r_{j} \xi}$ and $v(\xi)=\int_{[0,1]^{2}} \exp (i \lambda \xi \phi(x, y)) d x d y$. Since $\hat{h}$ is a Schwartz function, we thus have

$$
\left|\bigcup_{j} E_{j}\right| \leq C \int_{-\infty}^{\infty}(1+|\xi|)^{-2}|u(\xi) v(\xi)| d \xi
$$


Since the points $\lambda r_{j}$ are 1-separated, the standard orthogonality computation gives

$$
\int_{a}^{a+1}|u(\xi)|^{2} d \xi \leq C N
$$

uniformly in all $a \in \mathbb{R}, N \geq 1$. Also $|u(\xi)| \leq N$ for all $\xi$, while

$$
|v(\xi)| \leq C \min \left(1, \lambda^{-1 / 2}|\xi|^{-1 / 2}\right) .
$$

The contribution of the region $|\xi| \leq N^{-1}$ to the above integral is therefore at most

$$
C \int_{|\xi| \leq 1 / N} N \lambda^{-1 / 2}|\xi|^{-1 / 2} d \xi \leq C N^{1 / 2} \lambda^{-1 / 2}=C N^{1 / 2} \delta^{1 / 2} .
$$

The contribution of the region $N^{-1} \leq|\xi| \leq 1$ is bounded by

$$
C \lambda^{-1 / 2}\left(\int_{-1}^{1}|u(\xi)|^{2}\right)^{1 / 2} \cdot\left(\int_{N^{-1} \leq|\xi| \leq 1}|\xi|^{-1}\right)^{1 / 2} \leq C \delta^{1 / 2} N^{1 / 2} \sqrt{\log (1+N)} .
$$

For the contribution of any region $k \leq|\xi| \leq k+1$, where $k \geq 1$ is an integer, one obtains by the same reasoning a bound of $k^{-5 / 2} \delta^{1 / 2} N^{1 / 2}$. Summing over $k$ completes the proof.

Corollary 9.2. If $\phi$ satisfies the hypotheses of Proposition 9.1, then for every measurable subset $E \subset Q$ of positive Lebesgue measure, the image set $\phi(E) \subset \mathbb{R}^{1}$ has Hausdorff dimension one.

Proof. Suppose that $\phi(E)$ has Hausdorff dimension $D<1$; we aim to show that $|E|=0$. Then there exists $C<\infty$ such that for each small $h>0$ there exists a cover $\left\{B_{j}=B\left(x_{j}, r_{j}\right)\right\}$ of $\phi(E)$ by balls, with $\sum_{j} r_{j}^{D} \leq C$ and $r_{j}<h$ for all $j$. Fixing $h$ and such a cover, for each integer $k$ satisfying $2^{-k}<h$ we denote by $N_{k}$ the number of balls $B_{j}$ for which $r_{j} \in\left(2^{-k-1}, 2^{-k}\right]$, and by $E_{k}$ the set of all points in $E$ for which $\phi(x, y)$ lies in some ball $B_{j}$ with radius $r_{j} \in\left(2^{-k-1}, 2^{-k}\right]$. Then $\sum_{k} N_{k} 2^{-k D} \leq C$, so $N_{k} \leq C 2^{k D}$. By Proposition 9.1, $\left|E_{k}\right| \leq C 2^{-k / 2}\left(N_{k} \log \left(2+N_{k}\right)\right)^{1 / 2}$. Thus summing over all $2^{-k} \leq h$,

$$
|E| \leq \sum_{k}\left|E_{k}\right| \leq C \sum_{k} 2^{-k / 2}\left(2^{k D}(1+k)\right)^{1 / 2} \leq C h^{s}
$$

for all $s<(1-D) / 2$. Letting $h \rightarrow 0$ concludes the proof.

Similar reasoning gives a stronger conclusion in higher dimensions. If $\phi:[0,1]^{n} \times$ $[0,1]^{n} \mapsto \mathbb{R}$ is integrable with a $C^{\infty}$ mixed Hessian $\partial^{2} \phi / \partial x \partial y$ whose determinant vanishes nowhere, then there is a bound of $C(1+|\lambda|)^{-n / 2}$ for the oscillatory integrals. If $n>1$, this leads to the conclusion that for every measurable set $E$ of positive Lebesgue measure, $\phi(E)$ contains a set of positive measure.

\section{ACKNOWLEDGEMENT}

The authors would like to thank Stephen Wainger, without whose input this project would never have begun, let alone been finished. They also thank Professors D. H. Phong, A. Seeger and E. M. Stein for many conversations and discussions on these topics, and T. Tao for contributing to the remarks in $\S 9$. Finally, they acknowledge the hospitality of ICMS, Edinburgh, where this research began, and of MSRI, Berkeley, where it was concluded. 


\section{REFERENCES}

[AKC] G.I. Arhipov, A.A. Karacuba and V.N. Cubarikov, Trigonometric Integrals, Math. USSR Izvestija 15, 2 (1980) 211-239. MR 81f:10050

[CP] A. Carbery and S. Pérez Gómez, in preparation.

[CRW] A. Carbery, F. Ricci and J. Wright, Maximal functions and Hilbert transforms associated to polynomials, Rev. Mat. Iberoamericana 14 (1998), 117-144.

[CSWW] A. Carbery, A. Seeger, S. Wainger and J. Wright, Classes of singular integral operators along variable lines, to appear, J. Geom. Anal.

[CWW] A. Carbery, S. Wainger and J. Wright, Hilbert transforms and maximal functions associated to flat curves on the Heisenberg group, J. Amer. Math. Soc. 8 (1995), 141-179. MR 95g: 43010

[C1] M. Christ, Failure of an endpoint estimate for integrals along curves, in Fourier Analysis and Partial Differential Equations, pp 163-169, CRC Press, 1995. MR 97e:44007

[C2] M. Christ, Hilbert transforms along curves, I: Nilpotent groups, Annals of Math. 122, (1985), 575-596. MR 87f:42039a

[E] P. Erdös, On extremal problems of graphs and generalized graphs, Israel J. Math. 2 (1964), 183-190. MR 32:1134

[F] K. J. Falconer, The Geometry of Fractal Sets, Cambridge Univ. Press, Cambridge, 1985. MR 88d:28001

[H] L. Hörmander, Oscillatory integrals and multipliers on $F L^{p}$, Ark. Mat. 11 (1973), 1-11. MR 49:5674

[IK] E. Isaacson and H.B. Keller, Analysis of numerical methods, John Wiley, 1966. MR 34:924

[JS] W.B. Jurkat and G. Sampson, The complete solution to the $\left(L^{p}, L^{q}\right)$ mapping problem for a class of oscillatory kernels, Ind. U. Math. J. 30, 1, (1981), 403-413. MR 84i:42033

[K] N. Katz, Self crossing six sided figure problem, preprint.

[Kch] U. Keich, On $L^{p}$ bounds for Kakeya maximal functions and the Minkowski dimension in $\mathbf{R}^{2}$, Bull. London Math. Soc. 31 (1999), no. 2, 213-221. CMP 99:06

$[\mathrm{Ke}] \quad \mathrm{T}$. Keleti, Density and covering properties of intervals of $\mathbb{R}^{n}$, preprint.

[P] Y. Pan, Uniform Estimates for oscillatory integral operators, J. Funct. Anal. 100 (1991) 207-220. MR 93f:42034

[PS1] D.H. Phong and E.M. Stein, On a stopping process for oscillatory integrals, J. Geom. Anal. 4 (1994) 104-120. MR 95k:42025

[PS2] D.H. Phong and E.M. Stein, The Newton polyhedron and oscillatory integral operators, Acta. Math. 179 (1997) 105-152. MR 98j:42009

[RS] F. Ricci and E.M. Stein, Harmonic analysis and nilpotent groups and singular integrals I. Oscillatory integrals, J. Funct. Anal. 73 (1987), 179-194. MR 88g:42023

[R] W. Rudin, Real and Complex analysis, McGraw Hill, New York (1966). MR 35:1420

[Se1] A. Seeger, $L^{2}$ estimates for a class of singular oscillatory integrals, Math. Res. Lett. 1, (1994) 65-73. MR 95e:42005

[Se2] A. Seeger, Radon transforms and finite type conditions, J. Amer. Math. Soc. 11 (1998), no. 4, 869-897. MR 99f:58202

[Sj] P. Sjölin, Convolution with oscillating kernels, Ind. U. Math. J. 30 (1981), 47-55. MR 82d: 42018

[S] E.M. Stein, Harmonic Analysis, Princeton U. Press, Princeton, 1993. MR 95c:42002

Department of Mathematics \& Statistics, University of Edinburgh, King's Buildings, Edinburgh EH9 3JZ, Scotland, United Kingdom

E-mail address: carbery@maths.ed.ac.uk

Department of Mathematics, University of California, Berkeley, California 947203840

E-mail address: mchrist@math.berkeley.edu

Department of Mathematics, University of New South Wales, 2052 Sydney, New South Wales, Australia

E-mail address: jimw@maths.unsw.edu.au 